Article

\title{
Concrete Preliminary Damage Inspection by Classification of Terrestrial Laser Scanner Point Clouds through Systematic Threshold Definition
}

\author{
Zahra Hadavandsiri * ${ }^{-}$, Derek D. Lichti ${ }^{\circledR}$, Adam Jahraus and David Jarron \\ Geomatics Engineering Department, University of Calgary, Calgary, AB T2N 1N4, Canada; \\ ddlichti@ucalgary.ca (D.D.L.); adam.jahraus@lidarservices.ca (A.J.); dmjarron@ucalgary.ca (D.J.) \\ * Correspondence: zhadavan@ucalgary.ca; Tel.: +1-403-210-7140
}

Received: 25 October 2019; Accepted: 9 December 2019; Published: 13 December 2019

\begin{abstract}
This paper presents a novel approach for automatic, preliminary detection of damage in concrete structures using ground-based terrestrial laser scanners. The method is based on computation of defect-sensitive features such as the surface curvature, since the surface roughness changes strongly if an area is affected by damage. A robust version of principal component analysis (PCA) classification is proposed to distinguish between structural damage and outliers present in the laser scanning data. Numerical simulations were conducted to develop a systematic point-wise defect classifier that automatically diagnoses the location of superficial damage on the investigated region. The method provides a complete picture of the surface health of concrete structures. It has been tested on two real datasets: a concrete heritage aqueduct in Brooks, Alberta, Canada; and a civil pedestrian concrete structure. The experiment results demonstrate the validity and accuracy of the proposed systematic framework for detecting and localizing areas of damage as small as $1 \mathrm{~cm}$ or less.
\end{abstract}

Keywords: structural damage assessment; TLS; automatic damage classification; robust PCA; systematic threshold

\section{Introduction}

Concrete structures are subject to damage and material degradation over their lifetimes due to human activities and environmental and natural hazards [1]. It is crucial to detect and characterize the extent and seriousness of the damage on the surface of the concrete in order to assess the integrity and safety of the structure. Early detection of a potentially dangerous situation allows timely interventions and maintenance procedures that can improve the current condition and preserve the structure prior to costly repair or even catastrophic collapse, and could lead to a longer lifetime for the structure [2]. Therefore, development of suitable inspections is extremely important to achieve accurate and reliable surface diagnosis of the condition of the concrete structure $[3,4]$.

Based on the simple visual appearance, the type of concrete damage can be categorized as cracking, crazing, spalling, scaling, delamination, dusting, blistering, efflorescence, and discoloration [5]. Cracking is a path of the local separation of a structural element or material into two, or more, pieces. Crazing, also called pattern cracking or map cracking, is the formation of closely spaced shallow cracks in an uneven manner. Spalling can be described as the breaking of layers or pieces of concrete from the surface of a structural element. Concrete scales when the finished surface flakes or peels off. Delamination is when the top surface of the concrete becomes separated from underlying concrete and is similar to blistering. Dusting, also called chalking, is the formation of fine and loose powdered concrete on the hardened concrete by disintegration. Blistering is the formation of hollow bumps of different sizes on the concrete surface. Efflorescence is the formation of deposits of salts on the concrete 
surface. The salts formed are generally white in color. Discoloration of a concrete surface can appear as gross color changes in concrete as spotted or mottled light or dark blotches on the surface, or early light patches of efflorescence [5].

Damage assessment of historic concrete structures is critical for their preservation and sustainability. However, they pose technological challenges at different levels, from the selection of proper sensors to the design of a structural health evaluation paradigm. The complex behavior of these structures necessitates the use of reliable tools for the preservation and risk mitigation. Consequently, correct diagnosis of their health conditions is provided, and potential vulnerabilities are identified in order to prevent the risk of damage and to design adequate solutions in advance [6]. The inspection technologies available today have difficulty in detecting slowly progressive and locally limited damage, especially in hard-to-reach areas in the structure [7].

Traditional diagnostic methods of concrete damage assessment first carry out periodic visual inspections. Following the preliminary visual inspection, a more thorough field survey of the defects identified is required to enable detection of deep material pattern of anomalies. Non-destructive tests (NDT) might be required to evaluate the preliminary inspections and define an intervention plan [8]. Various NDT tests on concrete structures have been presented in the literature, including the penetration method, the rebound hammer test, the ultrasonic pulse velocity test and so on [9]. Structural damage can be detected using embedded sensors that detect structural stiffness changes or corrosion initiation, for example, vibrating wires [10] and fiber optic interferometric sensors [11] based on vibration tests, neural networks, wavelet transforms, and nonlinear finite element methods [12]. Despite very high accuracy (up to a few hundredths of a millimeter), these methods are time consuming, subject to human error, and capable of point wise measurements in one-dimension and thus can only evaluate a few discrete areas of the structure [13]. In the last few decades, high quality remote sensors have overcome the limitations associated with the traditional inspections [14].

Evidently, digital imaging and light detection and ranging (LiDAR) are considered as the best solutions for concrete preliminary damage detection due to their ability to offer high spatial resolution [15]. Consequently, damage occurring to any part of the structure can be detected and localized. For instance, Jenkins et al. [16] performed visual inspection and structural condition monitoring of railway tunnels based on imagery data and an image processing software package carrying out operations such as image registration and stitching, 3D reconstruction and change detection. Image-based health monitoring techniques to acquire 3D point clouds (PCs) on real scenes are low-cost and very precise, but the quality of the images is significantly affected by the surrounding environmental conditions and exhaustive images are needed to achieve the required resolution [17]. The recent development of robotic equipment such as unmanned aerial vehicles (UAVs) facilitates the acquisition of high-resolution aerial photos from which 3D PCs are obtained through Structure-from-Motion (SfM) [18] and MultiView-Stereo (MVS) photogrammetry algorithms [19,20]. Skarlatos and Kiparissi [21] showed that for small and medium size objects and distances, an SfM algorithm followed by an MVS algorithm is superior to terrestrial laser scanner (TLS) in terms of accuracy, density, modelling quality and cost. However, factors such as poor texture and weak network geometry may limit the effectiveness of SfM-MVS. In addition, they demonstrated that, for large-scale objects such as historic structures, a laser scanner is better in terms of quality and processing time. A single scan acquired with laser scanner technology can cover a full $360^{\circ} \times 310^{\circ}$ panoramic view and directly generate a dense 3D PC within few minutes with much higher precision and resolution [22].

In the last few years, much research has focused on automatic methods for structural deformation monitoring from remotely sensed data captured by TLS due to its ability to offer high spatial resolution at high speed and high accuracy [23,24]. Valença et al. [25] used geometric information surveyed by TLS for accurate image orthorectification, solving one of the major drawbacks of applying image processing for cracks characterization on large concrete bridges. Hancock et al. [26] studied change detection of fire-damaged concrete using TLS intensity data. 
For applications where the structure is not moving rapidly, a laser scanner having a sequential data collection scheme is capable of monitoring the structure over long-term time epochs. However, the change detection between pre- and post-event (the event might be an earthquake, although here, in the context of monitoring practice, event refers to the passage of time which causes damage to the structure) PC pairs derived from repeated TLS acquisition over the same area relies on the pre-event data, which is almost never available for old historic structures. Since these structures have not sustained damage within only a few years of this study, obtaining a temporal deformation monitoring, that is, the change detection between pre- and post-event PCs, is not applicable. Alternatively, instead of change detection on pre- and post-event PCs, damage assessment of the structure with respect to the design model or the idealized state of the structure will be the focus of this study.

This research solely uses the spatial TLS point cloud (PC) coordinates to localize geometric damage of any type which makes roughness as small as $1 \mathrm{~cm}$ or larger on a concrete surface. The synergistic application of deeper diagnostic techniques is essential to validate the identified surface damage. Therefore, complementary to the visual inspections facilitated by this research, concrete diagnostic procedures presently applied in the field of civil engineering such as NDT tests on the visually identified defects are required to enable inspection of the internal and deeper parts of the structure.

\section{Limitations of Previous Work}

Structural monitoring based on measuring temporal changes between PCs of different epochs, registered in a common coordinate system, has been well studied in the literature [27]. Due to the inability to exactly reposition a scanner at the same location and the finite laser beam footprint, individual measurements from repeated scans will not exactly coincide [28] and thus it is not possible to scan the same point in different epochs even if the scans are well registered. Therefore, temporal changes are mostly detected with respect to an object or at least a certain neighborhood of a point in the reference situation. By creating a 3D reference surface model from the points, rather than trying to detect deformation by single-point comparison, surface change is measured as the distance along the local surface normal $[29,30]$. Limitations of this approach include: (1) a specific surface model is needed for different applications with objects of specific geometric characteristics, Puente et al. [31] detected damage on planar masonry walls by measuring cloud-to-plane distances between repeated laser scanning data and were able to detect changes at the noise level of individual laser scanner observations while avoiding 3D point cloud registration; (2) when deformation is derived by computing the differences between surface models fitted to different epochs, the estimated deformation field is basically one-dimensional [32,33]; (3) cloud-to-mesh approaches do not take into account the local orientation of the surface represented by the PCs [27]; and (4) meshing TLS data not only adds a level of error by interpolation, but can also create erroneous surfaces [22]. Additionally, meshing PCs have severe limitations specially for sophisticated historic structures presenting roughness at all scales [34].

Barsanti et al. [35] evaluated a region-growing algorithm to group points into clusters based on the angular comparison between locally estimated surface normals. They concluded that working on PCs does not seem to be the most suitable approach for creating a 3D segmentation to analyze historic concrete structures. On the other hand, when they applied the segmentation directly on meshes, appropriate results were only produced with the manual process and not with a completely automatic approach. Lague et al. [36] measured surface changes via a direct comparison of PCs without meshing or gridding. The first step is surface normal estimation and orientation in $3 \mathrm{D}$ at a scale consistent with the local surface roughness. In the second step, the mean surface change is measured by computing the local distance between the PCs of two epochs along the normal surface direction, which tracks 3D variation in surface orientation. Precise registration allows in situ change detection down to $6 \mathrm{~mm}$ at $95 \%$ confidence over ranges of $50 \mathrm{~m}$, depending on the instrument used. The main drawback is that the deformation is one-dimensional, along the direction of surface normal. Additionally, if a change in surface orientation has occurred between two epochs, the average normal is considered. Consequently, the deformation direction will be incorrect [15]. 
It is clear that it is possible to detect deformations below the nominal single point accuracy (a few millimeters) if appropriate modelling in combination with statistical error propagation is used [29]. However, for various applications, feasibility studies on the limits of TLS in detecting the minimum deformation with a predefined probability are a critical task [22,27].

In this study, change detection looks for a binary answer: whether and where a scene has been damaged, and thus classifies points into classes damaged/non-damaged. Although using imagery for damage classification is an extensively researched topic, to the best of the authors' knowledge, only a few publications have reported damage detection based on classifying spatial 3D points. Therefore, damage classification of spatial TLS PCs is a new research area that has the potential to enhance the current practice of inspections in structural health monitoring.

Current methods of damage detection based on PC classification are, firstly, limited to detecting specific shapes of defects occurring on specific geometric surfaces, such as flat planar structural components and are vulnerable to scan parameters such as incidence angle. For instance, Kim et al. [37] used surface normal vectors for localization of spalling defects on a flat surface. Shallow spalling defects less than $3 \mathrm{~mm}$ deep could barely be detected even when the detectability of defects was enhanced by an optimal incidence angle. Secondly, current methods are highly dependent on appropriate threshold values and thus might not be able to correctly model construction errors and surface inhomogeneities [38]. Thirdly, some methods are based on dividing the point cloud into sub-areas, with a predefined size, that are designated as damaged or undamaged. This subdivision reduces the resolution of the detection [39]. Moreover, they mostly avoid data artefacts such as registration errors by using non-registered data captured from a single position and, thus, they do not apply robust statistical methods to deal with data artefacts [38]. Distinguishing between damage and outliers is very critical in PC damage assessment [40]. Therefore, a robust statistical method such as robust PCA is required to enable detection of smaller and more detailed damage. For instance, Tang et al. [41] attempted to suppress noise in TLS PCs while maintaining information about surface flatness and achieved detection of flatness defects as small as $3 \mathrm{~cm}$ across and $1 \mathrm{~mm}$ thick only on horizontal surfaces.

The outline of the work is as follows. A review of the research problems and proposed objectives addressing these problems are stated in Section 2, followed by methodologies and simulations for developing an automatic classification procedure in Section 3. Section 4 describes the initial experiments conducted to gain heuristic knowledge on the collected dataset. Furthermore, the initial experiment results and findings by numerical simulations are discussed in Section 5 . Conclusions and discussion of open problems are presented in Section 6.

\section{PC Processing for Damage Assessment}

PC processing and surface modelling methods can detect damage on the surface of a structure according to the surface flatness, smoothness, and roughness with respect to a reference surface simulating the intact condition of the structure [42]. In this regard, the methods in the literature are based on classifying damage at either the point or the segment level. Geometric features, as descriptors of the local neighborhood at each point, provide insight into the structural geometric condition and are therefore used to classify the points [43]. PCA is a popular tool for linear dimensionality reduction and is commonly used for feature extraction.

Santos et al. [44] demonstrated that Kernel PCA (KPCA) can reveal more complicated hidden structures of the data than linear PCA and can enhance separation of damaged from undamaged conditions in acceleration time-series data captured of an aluminum frame structure in laboratory conditions. The KPCA algorithm is an extension of the linear PCA that maps data into a high-dimensional space to model nonlinear patterns presented in the original observation space and provide better classification performance [45]. Although, KPCA—similarly to kernel-based learning methods such as support vector machines-can handle the nonlinear relationship between features and class labels, its performance depends on the choice of kernel function and kernel parameters. Cross-validation, the most commonly used method to obtain these optimal kernel 
variables, is time consuming for massive data sets such as PCs applied in this study [44,46]. This research will demonstrate how a robust version of the linear PCA can improve shallow surface damage classification of PCs by masking changes caused by data artefacts from changes caused by damage.

One of the main distinguishing factors of our method is the use of supervised classification versus unsupervised classification. Supervised classification for damage assessment requires a user to manually select labelled data (training data) in order to train a classifier. The number of training samples needed to produce an accurate classification increases as the number of features increases. The training time can thus create a significant limitation, which negatively impacts the efficiency. For these reasons, this paper focuses on unsupervised classification approaches.

\subsection{Statement of Problems}

As mentioned, methods of structural damage assessment from spatial point clouds are based on classifying points as either damaged or undamaged. In this context, a comprehensive solution to the automated classification of raw TLS PCs is still a complex and challenging task due to the following facts:

- Due to a lack of current knowledge about the best feature to be used for damage detection, as many geometric features as possible are exploited, which increases the computational burden of large point clouds in terms of memory consumption and processing time.

- The distinctiveness of geometric features greatly depends on the respective neighborhood encapsulating those 3D points that are used to extract the features. Thus, applying an optimal neighborhood size is essential to compute non-biased and distinctive geometric features to allow the most accurate discrimination between different classes.

- The neighborhood size depends on irregular and varying point density and the variety of types of objects/damage to be detected.

- Subjective, scene-dependent, and inconsistent similarity thresholds are used in most classification and segmentation algorithms that change for different datasets captured in different environments.

- Outliers exist in the PCs due to data artefacts such as edge effects caused by occlusions, and the features derived have proven to be sensitive to outlier.

Furthermore, particular to this research, an undamaged surface is only approximately smooth and presents a roughness related to the characteristics of gravel used in concrete production. In addition, their surfaces may be distorted compared to their designed shapes due to construction errors. Therefore, reference surface model fitting cannot correctly model such distortions.

\subsection{Automatic Damage Classification}

A framework was developed to analyze 3D point clouds obtained from a concrete structure in order to automatically assign each 3D point a semantic label indicating whether it is damaged or not and, furthermore, to perform an automated damage assessment on a structure of interest. The following are the objectives of this work that address the aforementioned limitations and problems:

- Selection of an adequate feature to distinguish between undamaged and damaged surfaces.

- Assessment of impact of neighborhood size, point density, damage size and artefacts on damage detection.

- Systematic definition of thresholds for robust PCA classification of damage.

To meet these objectives, an unsupervised classification algorithm has been implemented to detect damage locations within a historic structure.

\section{Methodologies}

The input to the algorithm is the TLS PCs Cartesian coordinates captured from the surface of a concrete structure. The most defect-sensitive feature(s) and the optimal neighborhood, appropriate for 
damage detection, are empirically selected. Robust PCA, with the user-selected free inlier parameter, identifies and excludes outlier points in the selected neighborhood in order to derive a robust computation of the selected feature. The simulated impact of the remaining random measurement errors on the feature derived is used to establish systematic thresholds for classification. Accordingly, each 3D point captured from the concrete structure is automatically assigned a semantic label determining whether it is damage or not.

\subsection{Proposed Neighborhood Selection}

The local geometry at each query point, arising from the spatial arrangement and distribution of 3D points within its local neighborhood, is used to classify the points. An optimal neighborhood is defined as the largest set of spatially proximal points that belong to the same object so that points lying on different surfaces are excluded [47]. Various strategies exist for searching the local neighborhood around a given point [48]. The search might compute the k-Nearest Neighbors (kNN) or a range search around a query point.

Range search neighbors are more suitable for points with homogeneous density. On the other hand, in a kNN search, the area of interest (AOI) is adopted according to the point density by using a bigger AOI in the areas of lower point density (i.e., higher noise) [49,50]. Since this research aims to detect fine details of a 3D structure, such as damage in the order of $1 \mathrm{~cm}$, the $\mathrm{kNN}$ is not appropriate. When the dimensions of the object/damage are very small, the kNN may include several distinct structures and provide erroneous feature descriptors, which in turn adversely affects the damage classification. Therefore, a search based on spatial range will be used for this research. Rather than focusing on an optimal range search for each individual point, an optimal range search across all points is selected. The selection of a single optimal scale allows a generalization between datasets, since variations in point density will be handled.

On the one hand, neighborhood size should be sufficiently large to average out the effects of noise and thus allow reliable estimation of geometric features even in presence of noise. On the other hand, neighborhood size should be sufficiently small to allow detection of fine details of the damaged structure, because in a large neighborhood, each individual 3D point will contribute less to the surface variation estimate. Based on the heuristic knowledge gained through the initial experiments performed on part of the first collected dataset, the local surface variation (normalized smallest eigenvalue), $C_{\lambda}$, is selected as the most defect-sensitive feature and the fixed range of $2.5 \mathrm{~cm}$ is selected as the optimal neighborhood. These selected values are assumed to be applicable for any other dataset and this assumption will be validated on PCs collected from a second concrete structure. Accordingly, in the absence of an optimal kNN search, which minimizes the impact of point density, the threshold definition will focus on finding an automated procedure for quantifying the impact of density in the applied fixed range search.

\subsection{A Systematic and Data-independent Criterion for Damage Detection}

This research aims to investigate the impacts of neighborhood size, damage size, point density, geometric feature threshold, instrument precision, random errors and outliers (e.g., registration errors and edge effects) based on a systematic and data-independent criterion for damage detection. First, robust PCA parameters will be analyzed and tuned based on the sensitivity of the damage detection algorithm to the outliers. Second, systematic similarity thresholds are defined by assessing the impact of point density and random errors on damage detection. The defined thresholds based on point density will be combined with the robust outlier detection strategy (i.e., robust PCA) to achieve more robust geometric features suitable for classification of damaged PCs.

\subsubsection{Robust PCA}

PC classification based on the classical estimate of the dispersion matrix is highly affected by the presence of outliers in the data [51]. Robust statistical methods can be used to reduce the effect 
of outliers in model parameter estimation. For fitting of shape primitives such as planes to the local structure, the outlier problem may be overcome by the RANdom SAmple Consensus (RANSAC) algorithm or one of its variants. However, to describe the local structure via eigenvalues and derived features without assuming a parametric model, a robust version of PCA is preferred to reduce the effect of noise. Robust PCA is able to detect more outliers compared to RANSAC [52]. The effectiveness of robust PCA for outlier detection is well-established in planar and linear surface estimation [53], as well as in improving the cylindrical axis estimation [54].

The robust PCA is defined as computing the eigenvalues and eigenvectors of a robust estimator of the dispersion (covariance) matrix. There are a number of robust multivariate dispersion estimators in the literature. In this paper, the deterministic-MCD (Det-MCD) robust dispersion estimator, proposed by Hubert et al. [51], is applied for non-parametric, multivariate outlier detection due to its efficiency and practicality over other robust estimators. The Det-MCD algorithm estimates the mean and covariance matrix of the outlier-free neighborhood points referred to as the robust center (location) and scatter, respectively. For each neighborhood, the Det-MCD estimator selects a subset of $\mathrm{h}$ points (inliers) out of $n$ points with the smallest covariance determinant. The value of $h$ is computed based on the inlier percentage, $\alpha$, selected by the user, where (1- $\alpha)$ measures the fraction of outliers the algorithm should resist. Robust PCA is expected to improve the classification results by analyzing the sensitivity of damage detection to noise and thus applying appropriate $\alpha$ or $\mathrm{h}$.

\subsubsection{Automatic Definition of Thresholds for Robust PCA Classification}

Noisy point coordinates impact the precision of the derived eigenvalues and the respective features (e.g., normalized smallest eigenvalues). Although robust PCA reduces the influence of outliers on the eigenvalues derived, the variance of the eigenvalues due to remaining random measurement errors should be quantified. Therefore, the defined threshold must be large enough to incorporate the influence of random measurement errors on the derived features. Based on the law of variance propagation, the precision of the eigenvalues and the respective features can be theoretically derived from noisy point coordinates. To this end, the functional model is the formula for the 3D structure tensor from which the eigenvalues are estimated. The stochastic properties are the instrumental measurement random errors for the TLS instrument used to collect PCs in this study. Some research has attempted to analytically derive the impact of point errors on the principal components [43]. However, the exact theoretical derivations when point measurement errors have correlations (i.e., their covariance matrix contain non-zero off-diagonal elements) have not been derived.

In this research, Monte Carlo simulation was performed to simulate variations of the eigenvalues subject to random point measurement errors. The simulated impact of random point measurement errors on the eigenvalues can be used to establish systematic thresholds for classification. This in turn yields an automatic classification procedure. Based on the defined threshold for the adequate geometric features, the points will be classified as damage and non-damage.

\subsection{Statistical Behaviour and Distribution of the Smallest Eigenvalues of Wishart Covariance Matrices}

Let $X$ be the ensemble of real rectangular $\mathrm{n} \times \mathrm{p}(n \geq \mathrm{p})$ matrices containing $\mathrm{n}$ observations $x_{i}$ of a p-dimensional (e.g., $\mathrm{p}=3$ for 3D PCs) row vector drawn from a Gaussian distribution. It can be assumed that $\mathrm{X}$ has zero empirical mean without loss of generality by constructing new data as,

$$
X=X-\bar{X}, X \sim N_{\mathrm{n} \times \mathrm{p}}(0, \Sigma)
$$

Let $S_{n}=\frac{1}{n} X^{\prime} X \in \mathbf{R}^{\mathrm{p} \times \mathrm{p}}$ be a covariance matrix having ordered eigenvalues $\lambda_{1} \geq \lambda_{2} \geq \ldots \geq \lambda_{p}$ which are non-negative and are the squares of the singular values of $X$. By singular value decomposition, we can factorize $S_{n}$ with eigenvalues $\lambda_{j}$ in the diagonal matrix $L$ and $\left\{u_{j}\right\}$ are the orthogonal eigenvectors arranged as the columns of $U$,

$$
S_{n}=U L U^{\prime}=\Sigma \lambda_{j} u_{j} u_{j}^{\prime}
$$


The ensemble of $p \times p$ random symmetric covariance matrices, $M=X^{\prime} X$, are called Wishart covariance matrices and have a Wishart distribution with scalar matrix $\Sigma$ and with $n$ degrees of freedom. This is denoted by $M \sim W_{\mathrm{p}}(n, \Sigma)$ or $M \sim W(p, \mathrm{n})$. The singular value statistics of $X$ are completely determined by the eigenvalue statistics of the Wishart covariance matrices, $M=X^{\prime} X$. In fact, the ensemble of Wishart matrices, $M$, fluctuates around the empirical covariance matrix $S_{n}[55,56]$.

This research is particularly focused on the marginal density of the normalized smallest eigenvalues of Wishart covariance matrices constructed from rectangular random matrices of $X$ belonging to Gaussian ensembles. The smallest eigenvalue of the Wishart covariance matrices, $\frac{1}{n} X^{\prime} X$, or, equivalently, of $X^{\prime} X$, is of considerable interest for statistical analysis in many applications. In PCA, this smallest eigenvalue determines the best fit plane. This smallest eigenvalue is most sensitive to noise in the data and it gives the leading contribution for the threshold estimate [57].

The distributions of the extreme eigenvalues of Wishart matrices have been known in terms of zonal polynomials and in terms of Pfaffians [58,59]. Closed form expressions were not reported in the literature [60] until Edelman [61] integrated the joint eigenvalue distribution of real Wishart matrices and obtained a closed form for this integral, which is the exact probability density function (pdf) of their smallest eigenvalue. Edelman [61] only gave exact expressions for pdf of the smallest eigenvalue when rectangularity, $n-p$, equals to $0,1,2$, and 3 . Then, he used the first two of these expressions as terminal cases in a recursion to compute the distribution in the general case (for other values of rectangularity). He derived the pdf using recursion formulas that determine the polynomials for either even or odd rectangularity. Wirtz et al. [62] derived explicit closed form solutions for the smallest eigenvalue distribution in terms of Pfaffian determinants for both finite and infinite data matrix sizes in more compact and easier to handle form than the previously known complicated recursive expressions.

Despite the fact that the real ensembles are more versatile than their complex counterparts, real ensembles are technically challenging, particularly when correlations among the matrix elements are introduced. This can be proved using the law of variance propagation [63]. Although the spherical laser scanning measurement errors have an uncorrelated Gaussian distribution, their propagation into the Cartesian point coordinates are still Gaussian but correlated (i.e., the Wishart covariance matrices, $X^{\prime} X$, contain off-diagonal elements). Wirtz et al. [62] considered the uncorrelated Wishart model. In numerical simulations they include the correlated Wishart case and argue that for some kinds of correlated Wishart ensembles the smallest eigenvalue of the correlated model follows the same universal predictions. The numerical simulations underline that correlations in some kinds of correlated Wishart ensembles have a very weak effect on the spectral statistics of the smallest eigenvalues. However, they emphasize that they have not looked at the situation where covariance matrix develops a spectrum where some eigenvalues lie on the scale $1 / p$. Nor have they looked at the situation of doubly correlated Wishart ensembles [62].

In summary, covariance matrices constructed from rectangular random matrices belonging to Gaussian ensembles, have a Wishart distribution. Closed form solutions for the density of the smallest eigenvalues of uncorrelated Wishart matrices have been vastly studied in the literature. However, closed form solutions for density of the smallest eigenvalues of correlated Wishart matrices, applied in this study, have not been addressed in the literature. Therefore, this study approximates the statistical distribution that best fits the data set.

\subsection{Simulation Description}

The simulated uncertainties of the normalized smallest eigenvalue $\left(C_{\lambda}\right)$, selected as a distinctive geometric feature for the application of damage detection, can be used to systematically quantify the similarity thresholds based on point density. Based on the threshold defined for $C_{\lambda}$, the points will be classified as damage and non-damage.

A large number of random planes with different orientations and different distances from the origin ( $2 \mathrm{~m}$ to $28 \mathrm{~m}$ ) are simulated. The Cartesian coordinates of each point locating on these planes are calculated by projecting rays in vertical and horizontal angular increments according to the angular 
resolution of the TLS instrument used in this study. Each ray is then intersected with each plane to derive the range of the noise-free observations. The spherical inputs (i.e., vertical and horizontal angles and range) are contaminated with 100 trials of random instrumental measurement errors. The spherical random measurement precision obtained from the manufacturer's specifications, are assumed to be normally distributed. Next, the features are estimated with the $2.5 \mathrm{~cm}$ spherical neighborhood for a query point located at center of each simulated plane.

As shown in Figure 1, density varies inherently with changes of range and incidence angle in the simulated planes-as range and incidence angle increase, density decreases. Accordingly, the number of points, $n$, encapsulated in the applied fixed range search of $2.5 \mathrm{~cm}$ across the entire PCs, is an indicator of density variation among diversely oriented planes. Therefore, each simulated plane corresponds to an $n$ value indicating a specific density.

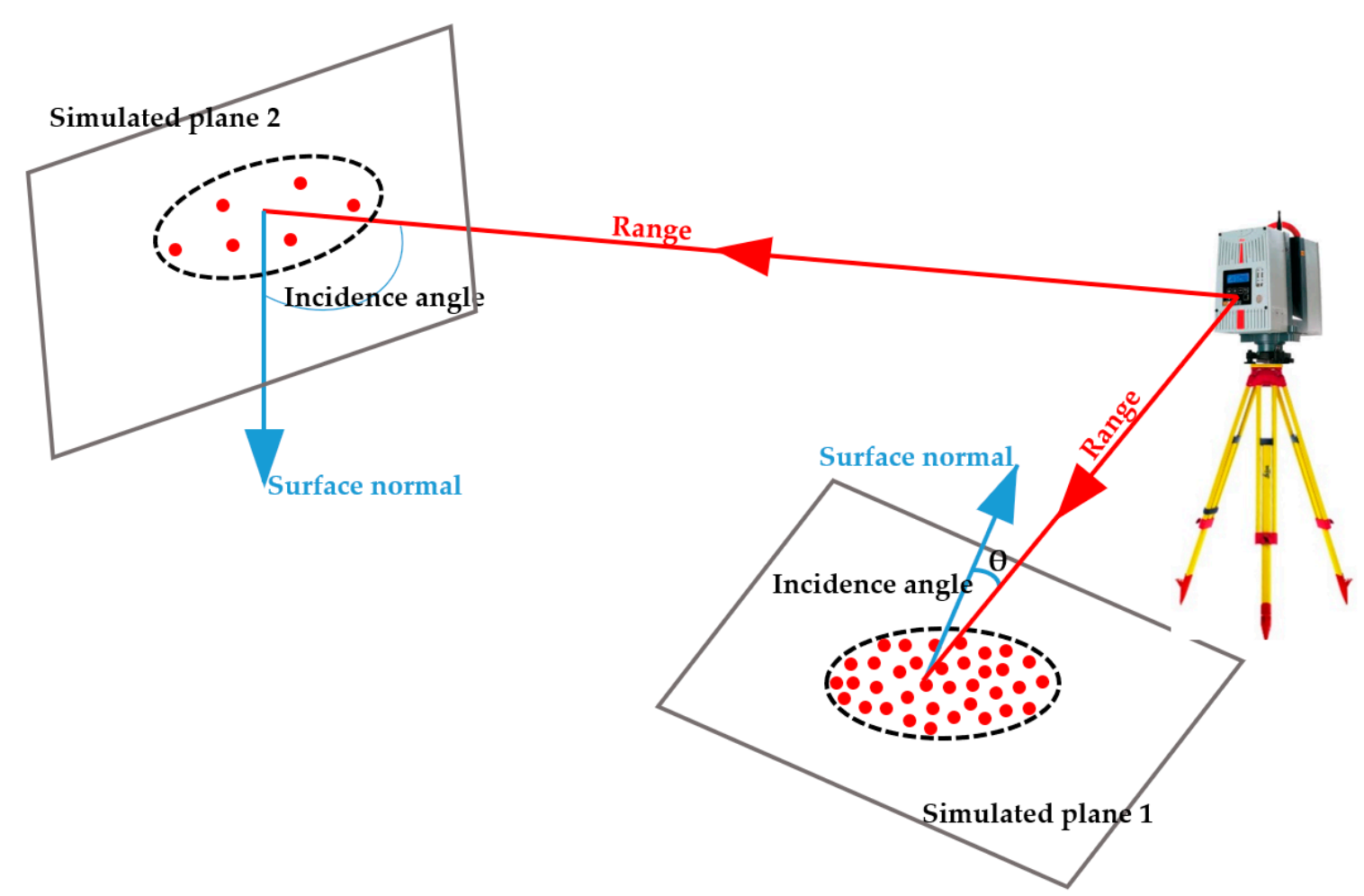

Figure 1. The query point at center of simulated plane 1 with smaller range and smaller incidence angle has larger $n$ in the fixed neighborhood of $2.5 \mathrm{~cm}$ (i.e., higher density) while simulated plane 2 corresponds to a smaller $n$.

The error-free normalized smallest eigenvalue, $C_{\lambda}$, should be zero for non-damaged planar points. Figure 2 illustrates the deviations of $C_{\lambda}$ from zero when the non-damaged planar points are contaminated with random errors across diversely oriented planes simulating various density values, $n$. Many candidate distributions were tested to find a statistical distribution that best fits to the $C_{\lambda}$ deviations at each cross section (e.g., the cross section shown in Figure 2) corresponding to a density value, $n$. 


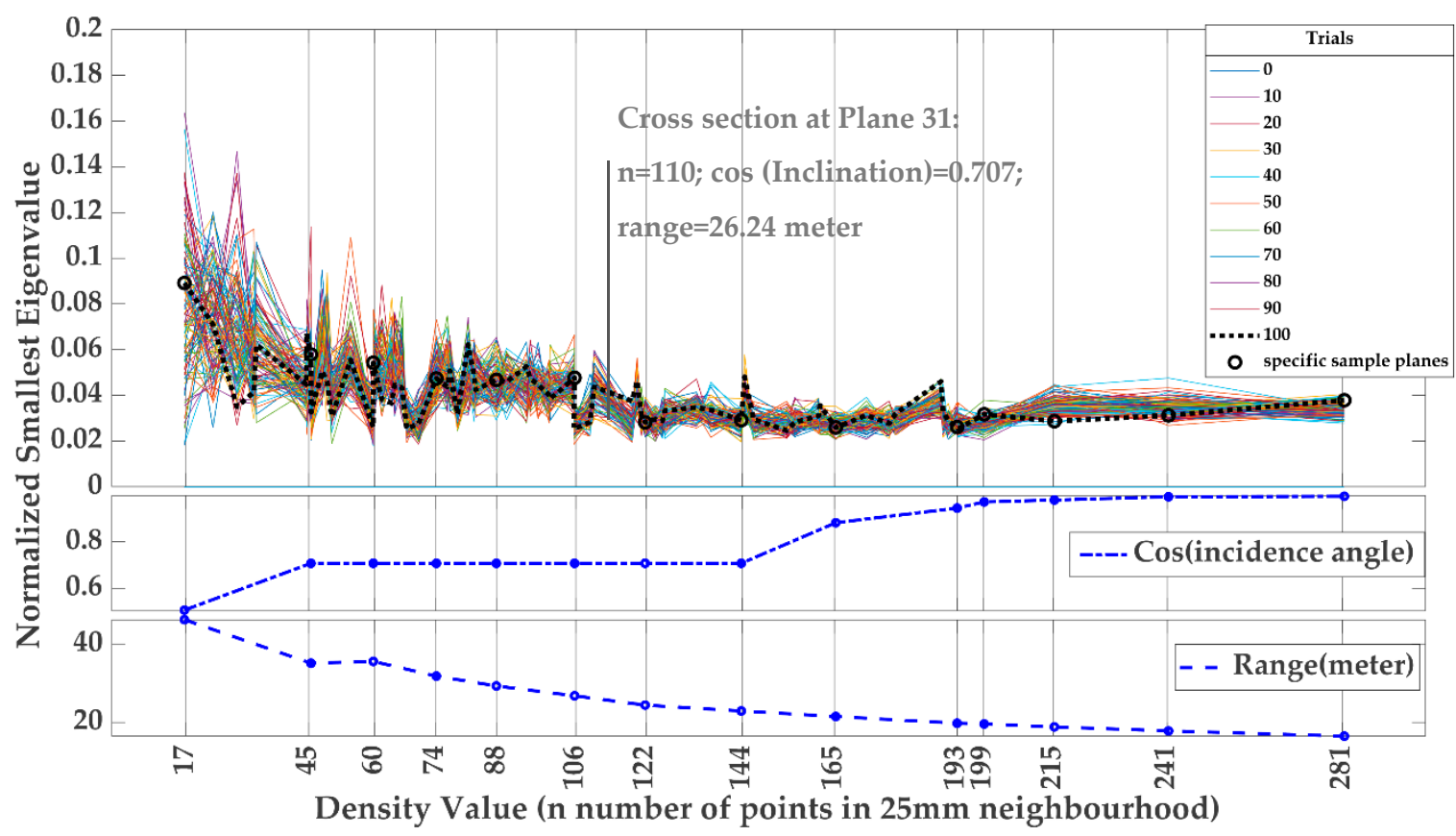

Figure 2. Deviations of $C_{\lambda}$ across diversely oriented planes for different trials.

The MATLAB probability distribution app was tested on several histograms corresponding to various density values. The Burr distribution was selected since it had the best fit in terms of minimum sum of squares of residuals. Figure 3 shows the Burr statistical distribution fitted to the data from a randomly selected plane shown in Figure 2.

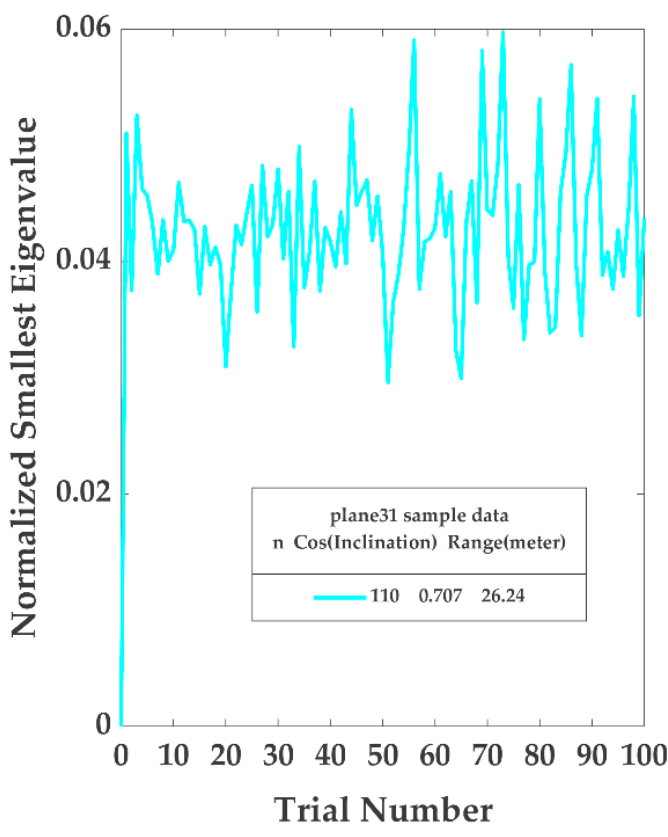

(a)

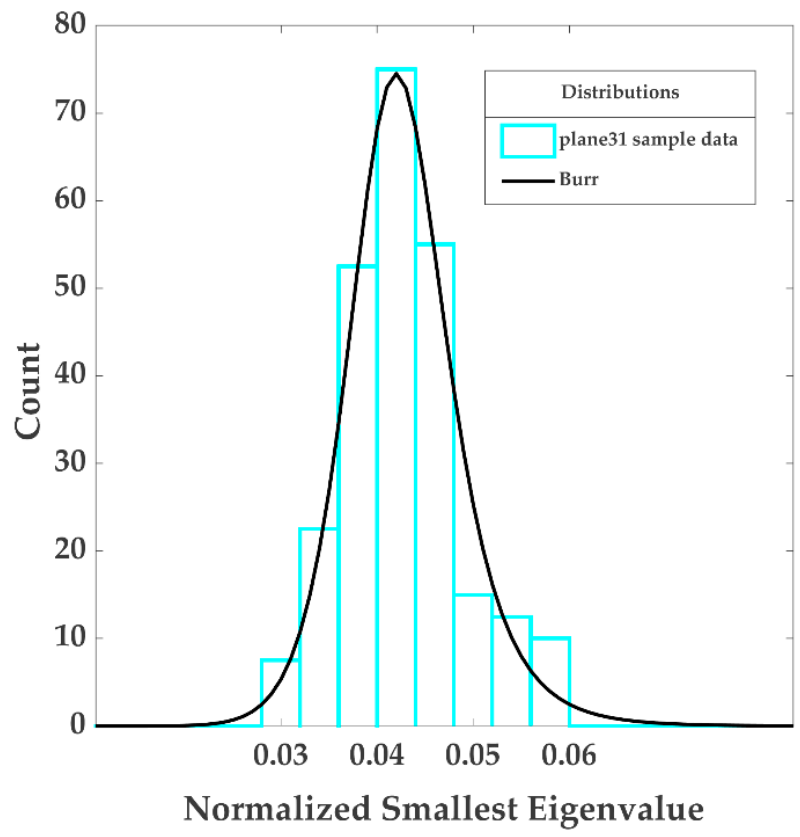

(b)

Figure 3. (a) deviations of $C_{\lambda}$ at the cross section of Figure 2; (b) histogram and Burr distribution fitted to data samples shown in (a). 
The Burr Type XII distribution, or simply the Burr distribution is a continuous parametric probability distribution for a non-negative random variable. The Burr probability density function (pdf) is

$$
f(x \mid a, c, k)=\frac{\frac{k c}{\mathfrak{a}}\left(\frac{x}{\mathfrak{a}}\right)^{c-1}}{\left(1+\left(\frac{x}{\mathfrak{a}}\right)^{c}\right)^{k+1}} \quad x>0, \mathfrak{a}>0, c>0, k>0
$$

where $c$ and $k$ are the first and second shape parameters and $\alpha$ is the scale parameter. Maximum likelihood estimation is used to determine the distribution parameters from the empirical sample data. The density of the Burr type XII distribution is L-shaped if $\mathrm{c} \leq 1$ and unimodal otherwise. For the Burr distribution fitted to the smallest eigenvalues, the shape parameter, $c$, is always larger than 1 , for any density value, $n$. Thus, the distribution increases at first, rising to a single clear peak or most frequent value, after which it decreases.

The Burr statistical distribution best fit to the data set generated by the deviations of $C_{\lambda}$ from zero across 100 random error trials describe the dataset at each density value, $\boldsymbol{n}$. Given the distribution of $C_{\lambda}$ for each density value, the 95th percentile value of the $C_{\lambda}$ distribution is defined as the simulated threshold value. Therefore, the defined threshold value is a function of density value, $n$. If the derived value of $C_{\lambda}$ is larger than the 95th percentile value (the critical value at the $5 \%$ significance level), the point is classified as damage with the probability of 95 percent.

Figure 4 shows Burr pdfs fit to the deviations of $C_{\lambda}$ over different trials for each plane orientation simulating various densities. According to Edelman $[60,61]$, the distribution of the smallest eigenvalues gets wider as $n$ gets smaller, which agrees with Figure 4 . This is rational, since the smaller number of points in the fixed range search, $n$, indicates lower density which is less tolerant to the noise resulting in higher uncertainty of $C_{\lambda}$. In other words, the defined threshold value automatically quantifies the impact of point density variations in the applied fixed range search.

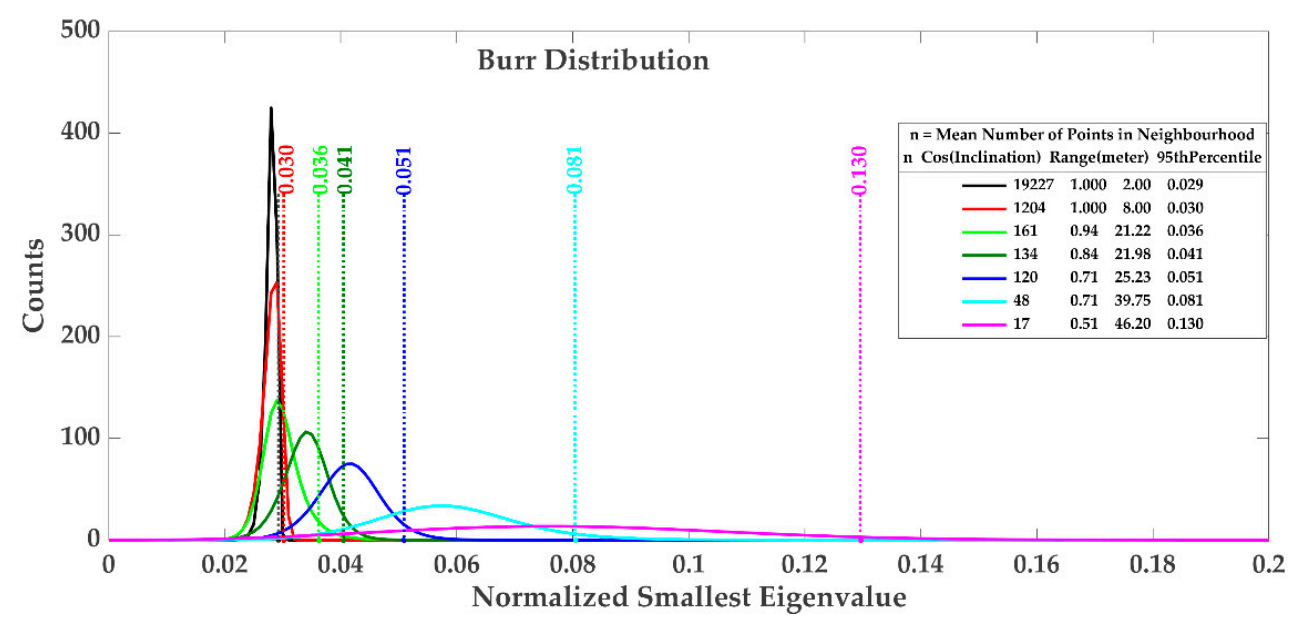

Figure 4. Burr distributions of $C_{\lambda}$ over different trials at diversely oriented planes having various density.

A large number of planes at different orientations producing various density values were simulated. For each density value, the 95 th percentile value of $C_{\lambda}$ distribution (i.e., where $95.45 \%$ of the $C_{\lambda}$ deviated values lie within two standard deviations of the mean value of $C_{\lambda}$ ), was plotted as the simulated threshold value on Figure 5. A curve fitted to the simulated threshold values quantified the systematic threshold as a function of density value. An exponential function of the form

$$
\text { Systematic Threshold }=\mathrm{f}(n)=n^{b}+d,
$$

was fitted at 0.95 confidence level. 


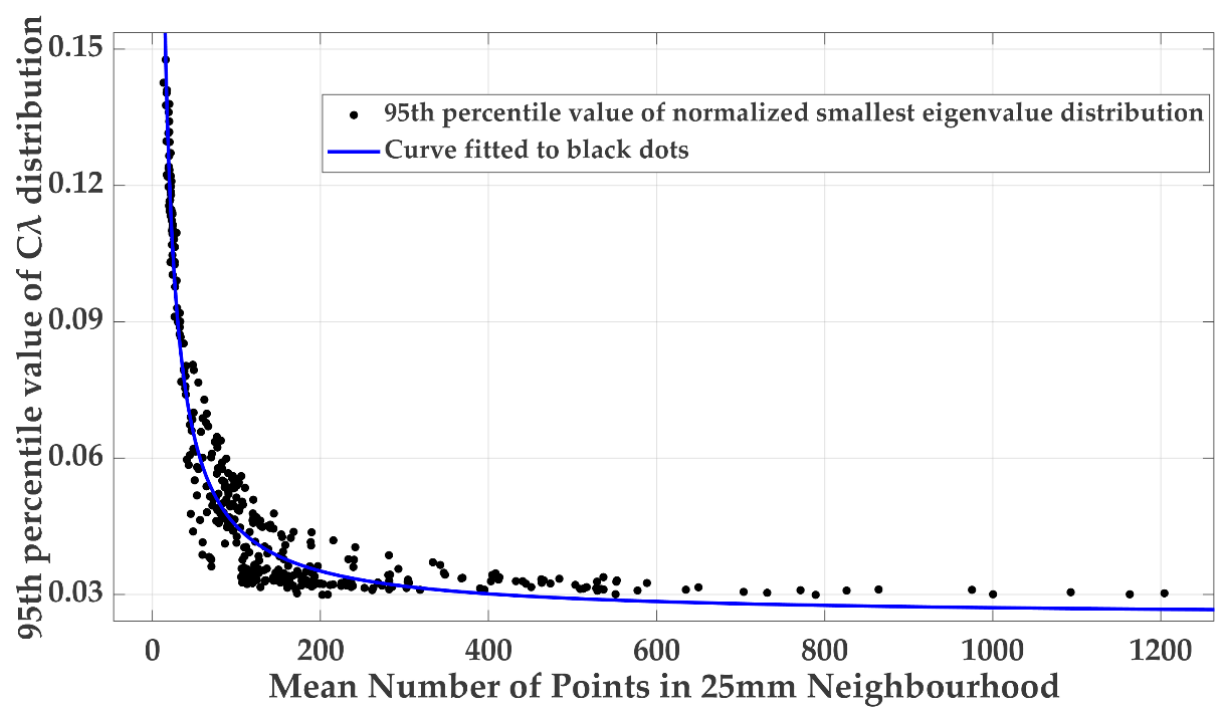

Figure 5. Exponential curve fitted to the simulated thresholds.

\section{Applications and Analysis}

\subsection{Experiment Environment}

The feasibility of the proposed damage assessment algorithm has been evaluated on data collected from a cultural heritage structure, an aqueduct site in Brooks, Alberta, Canada. The Brooks aqueduct (Figure 6), commissioned in 1914, is located in a shallow valley $5 \mathrm{~km}$ southeast of Brooks. The aqueduct is an impressive reinforced concrete structure over $3.1 \mathrm{~km}$ in length featuring a large catenary-shaped flume mounted on $20 \mathrm{~m}$ high columns [64].

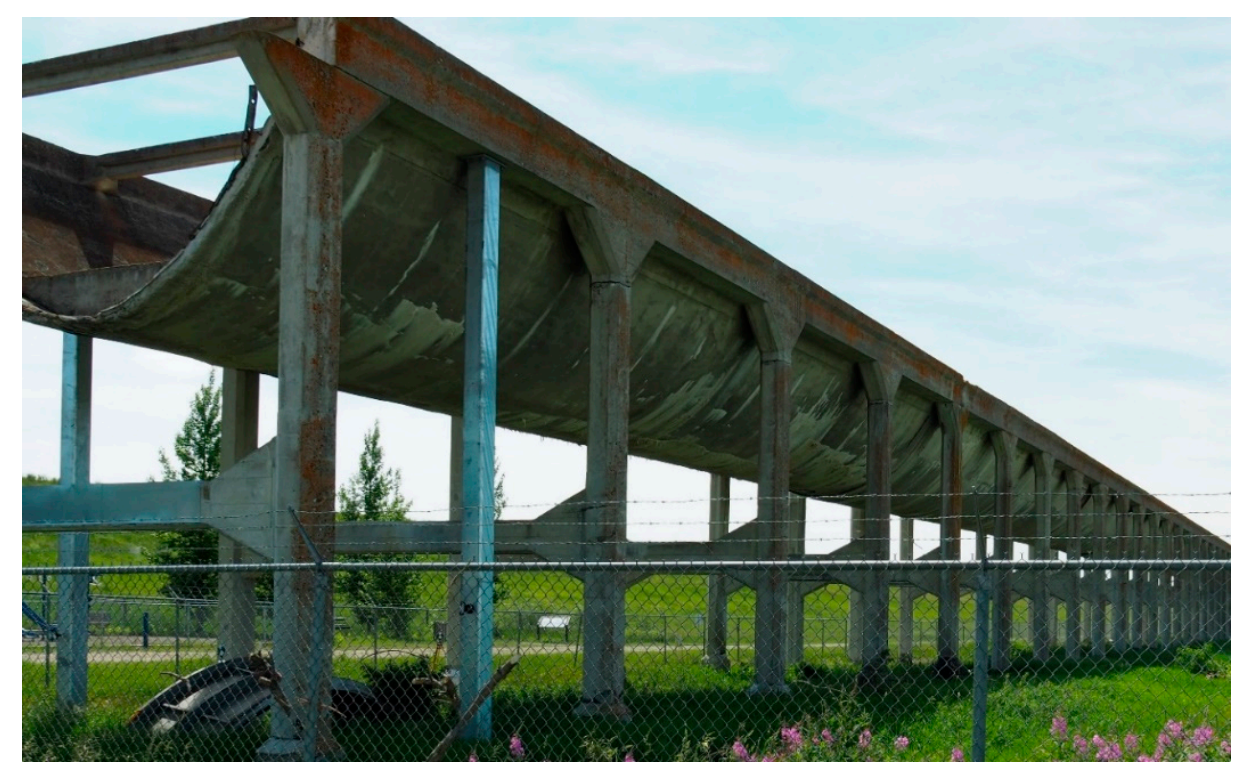

Figure 6. Brooks aqueduct.

The most unusual aspect of the aqueduct is the shape of the flume cross section, called a "hydrostatic catenary" by Muckleston and Gibb, and chosen because of its similarity to the hydraulically efficient semicircular cross section [64]. The flume part of the aqueduct is very elongated, which made it possible to analyze a complete range of scan distances, from $1 \mathrm{~m}$ to $100 \mathrm{~m}$, and scan beam incidence angles, from almost perpendicular to almost horizontal. 
The Brooks aqueduct was one of major water management facilities within the Eastern Section of southern Alberta. In 1979, the Brooks Aqueduct was abandoned with the completion of its earth fill replacement [64]. The aqueduct is a high maintenance structure [65]. Some instances of damage to the aqueduct concrete surface are visible to human eyes, and have been documented as digital photo images, thus, damage assessment of the structure is vital for preservation.

\section{Data Preparation}

The site was scanned by Leica Geosystems High-Definition Surveying (HDS) 6100 laser scanner with scanning settings summarized in Table 1 . The HDS 6100 has a rotating mirror system that covers a $360^{\circ} \times 310^{\circ}$ field of view. In addition to the spatial location of the reflecting surface point, the intensity of the received echo is recorded.

Table 1. Leica High-Definition Surveying (HDS) 6100 scanning settings and manufacturer specifications ${ }^{1}$.

\begin{tabular}{cc}
\hline Resolution Level & Ultra High \\
\hline Angle increments horizontal & $0.009^{\circ}$ \\
Angle increments vertical & $0.009^{\circ}$ \\
Recommended target distance at angle of incident approx. 90 & $1-25 \mathrm{~m}$ \\
Maximum target distance & $30 \mathrm{~m}$ \\
\hline System Performance & Up to $50 \mathrm{~m}$ \\
Distance & $\leq 3 \mathrm{~mm} \mathrm{@} 90 \%$ albedo \\
Up to 25 m & $\leq 5$ mm @ $18 \%$ albedo \\
$\leq 3$ mm @ $18 \%$ albedo & Accuracy \\
Angle & $125 \mu$ rad \\
Horizontal & $125 \mu \mathrm{rad}$
\end{tabular}

${ }^{1}$ According to Leica HDS 6100 User Manual.

The dataset features a large variety of structures of various sizes as well as sparse vegetation on the ground. The point density slightly varies with the orientation of the surfaces with respect to the scanner position. The aqueduct 3D structure could not be completely captured from a single scan due its large dimensions, self-occlusions and occlusions by other objects. Thus, multiple scans were acquired from several positions with significant overlap to provide complete site coverage. The western part of the aqueduct was scanned from 12 scanner locations and the eastern part was scanned from 10 locations. For both western and eastern parts Leica black and white planar targets were distributed on and around the aqueduct columns to register the scans. The targets were fixed and not re-setup. The registration, performed in Cyclone Leica Geosystems in unlevelled and target-based mode, resulted in 1-6 mm error between targets, while the root mean square (RMS) error was 1-2 mm between common points in overlapping sections between scans. After removing the vegetation in the scene, iterative closest point (ICP) [66] was performed in Cloud Compare (CC), open source software (http://www.danielgm.net/cc, 2018), to further refine the registration.

\subsection{Initial Experiment}

An initial experiment was designed using part of the data set collected around visibly damaged areas to gain some heuristic knowledge specific to the dataset. The first column and the flume of the south facing side of the western part of the aqueduct were used as a test set (Figure 7). The test was performed on the top, middle, and bottom of the column, as well as its connected flume. The test objective was to examine the relation between density, damage size, neighborhood size, and geometric feature threshold. As a result, the optimal combination of the variables mentioned that yields the most accurate discrimination between different classes, (i.e., being able to detect damage), while improving computational efficiency in terms of both time and memory consumption, could be found. 


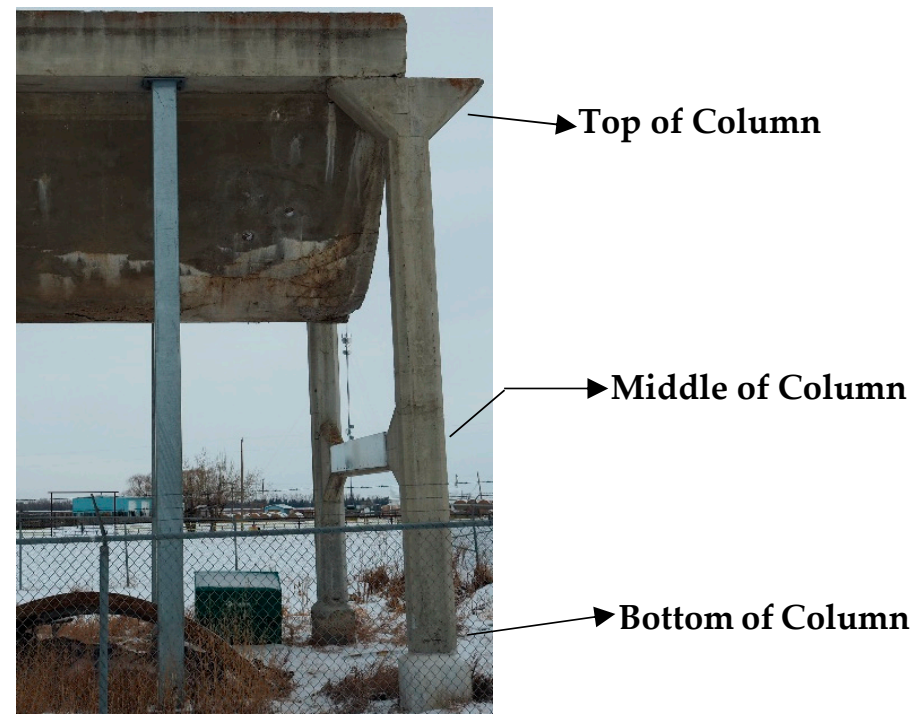

Figure 7. The first column and flume, south facing of the western part of the aqueduct.

For now, instead of focusing on an optimal 3D neighborhood for each individual point, a global optimal neighborhood size was found. To this end, a fixed range search was varied and a specific geometric feature was calculated at each scale. In this regard, the abovementioned variables were examined:

- Geometric feature: based on the priori knowledge that the aqueduct's 3D structure can be locally modelled as a planar feature while damage can be detected based on the smoothness of surface, normalized smallest eigenvalue, $C_{\lambda}$, was selected as an adequate and distinctive geometric feature. Since the eigenvector corresponding to the $\lambda_{\min }$ points in the direction normal to the plane, planar points exhibit a small $C_{\lambda}$, whereas damaged surfaces exhibit a large $C_{\lambda}$.

- Neighborhood size: the test started with a small range search that was successively increased. The interval between range $e_{\min }=1 \mathrm{~cm}$ and range $\max =5 \mathrm{~cm}$ was sampled in scales, $1 \mathrm{~cm}, 1.5 \mathrm{~cm}$, $2 \mathrm{~cm}, 2.5 \mathrm{~cm}, 3 \mathrm{~cm}$, and $5 \mathrm{~cm}$ (Figure 8). $C_{\lambda}$ was examined over a range of thresholds including $0.2,0.1,0.05,0.04,0.03,0.02$, and 0.01 . A critical neighborhood size corresponds to a significant increase of $C_{\lambda}$, so that, planar points are erroneously detected as damage.

- Density: data were tested at the original, $1 \mathrm{~mm}$, and down sampled to $5 \mathrm{~mm}$ scanning resolution of PCs to ensure the minimum density required for detecting damage. The collected point cloud was down sampled by reducing the minimum spacing between points from original state to $5 \mathrm{~mm}$.

- Damage size/different objects: the test set contains a large variety of structures and damage of various sizes.

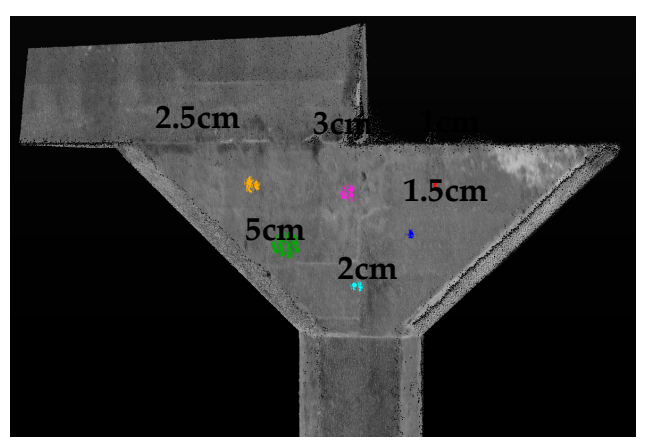

(a)

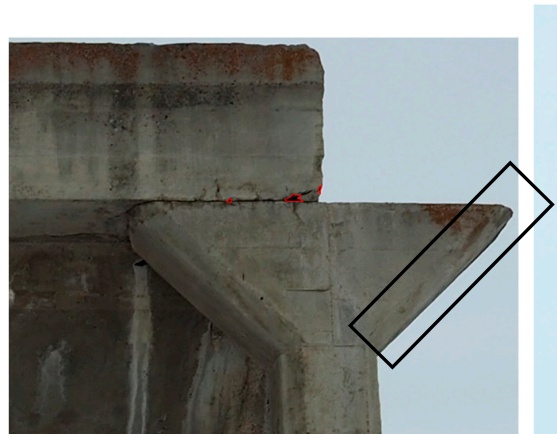

(b)

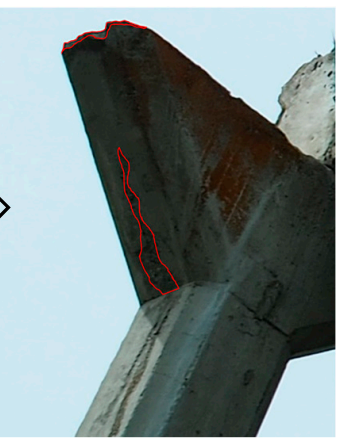

(c)

Figure 8. (a) Range scales sampling damage shown on top of the column, south facing of the western part of the aqueduct; (b) is the corresponding digital image; (c) is different perspective view of the rectangle area in (b). 
As the outcome of testing the above variables, heuristic knowledge of the optimal combination of the variables mentioned, which yields the most accurate discrimination between different classes, was found for the test data that is described in the next section. It is assumed that this heuristic knowledge could be applicable to any other dataset. This assumption will be validated on PCs collected from a second concrete structure.

\section{Results and Discussions}

\subsection{Initial Experiment results}

Areas of damage were manually delineated on the imagery in red for validating the relative position of the corresponding defective areas identified from the scans (shown in Figure 8, Figures 10-12, 18, 19, 21, 22). The PC results are visualized in CC as follows: linear points in green; planar points in red; and the non-smooth points in blue. Therefore, damage is expected to be shown in blue. Linear points represent points at edges and points at the boundary of a cropped area of the structure. The classical PCA test results show that at $5 \mathrm{~mm}$ resolution, $2 \mathrm{~cm}$ or $2.5 \mathrm{~cm}$ neighborhood size and $C_{\lambda}$ thresholds of $0.04,0.03$, or 0.02 , the most accurate discrimination between damage and non-damage classes is provided. Figures 9-12 illustrate classical PCA test results at $2.5 \mathrm{~cm}$ neighborhood size and $C_{\lambda}$ thresholds of 0.02 .

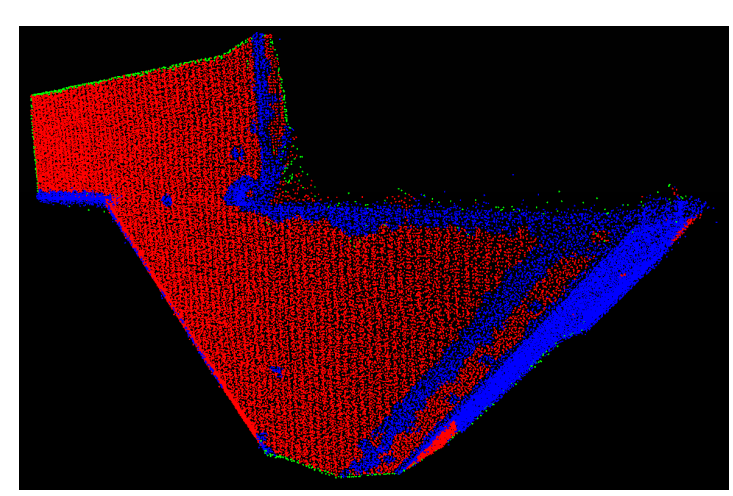

(a)

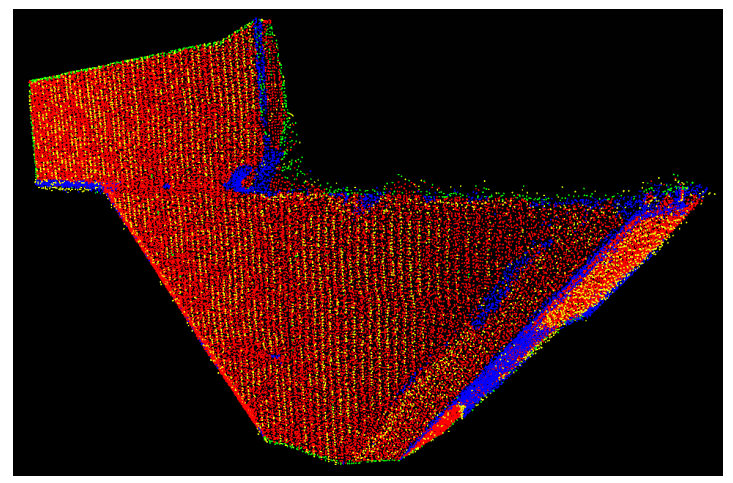

(b)

Figure 9. Top of the column. $5 \mathrm{~mm}$ point spacing, $C_{\lambda}$ thresholds of 0.02 , neighborhood size $2.5 \mathrm{~cm}$. (a) classical principal component analysis (PCA); (b) robust PCA at $\alpha=0.75$.

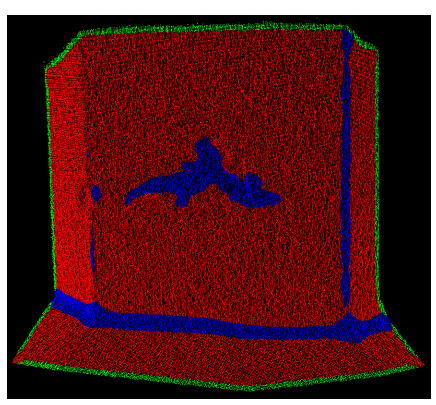

(a)

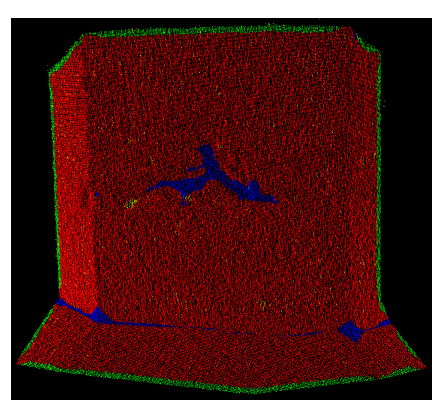

(b)

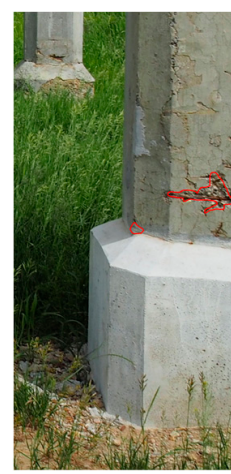

(c)

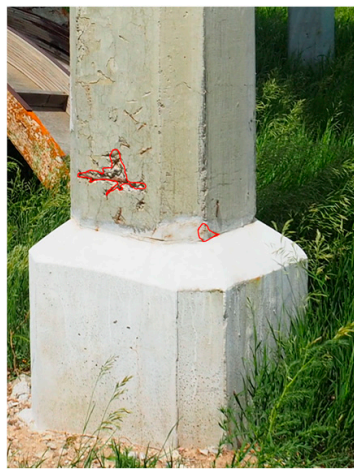

(d)

Figure 10. Bottom of the column. $5 \mathrm{~mm}$ point spacing, $C_{\lambda}<0.02$, neighbourhood size $2.5 \mathrm{~cm}$. (a) classical PCA; (b) robust PCA at $\alpha=0.75$. (c) and (d) are the corresponding digital images. 


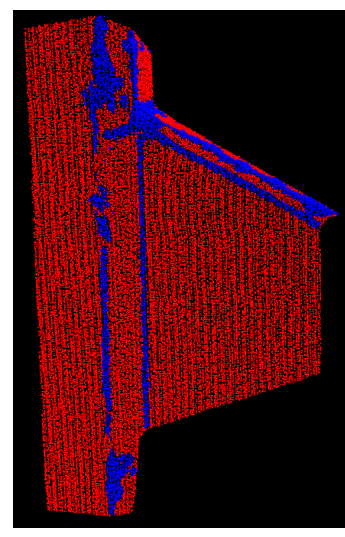

(a)

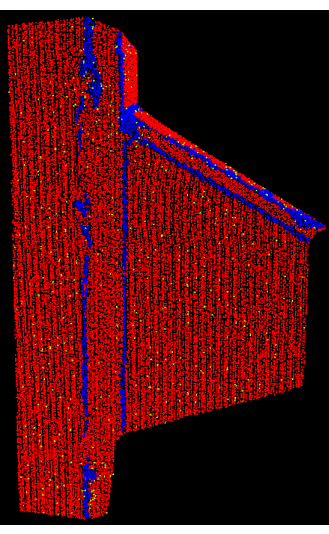

(b)

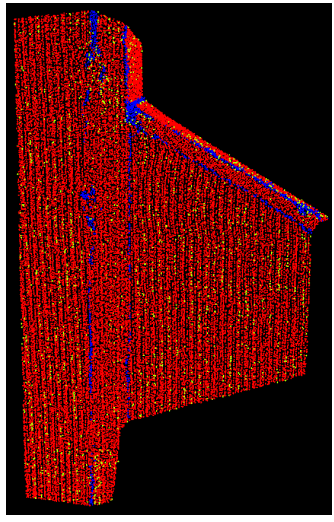

(c)

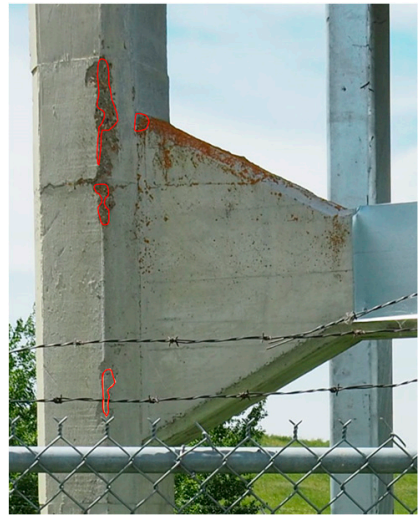

(d)

Figure 11. Middle of the column. $5 \mathrm{~mm}$ point spacing, $C_{\lambda}<0.02$, neighbourhood size $2.5 \mathrm{~cm}$. (a) classical PCA; (b) robust PCA at $\alpha=0.90 ;(\mathbf{c})$ robust PCA at $\alpha=0.75 ;(\mathbf{d})$ is the corresponding digital image.

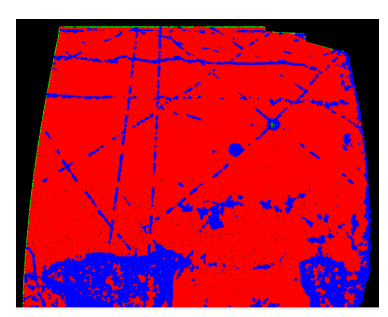

(a)

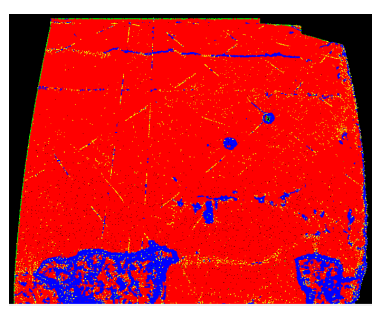

(b)

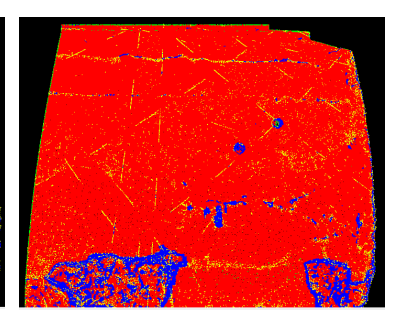

(c)

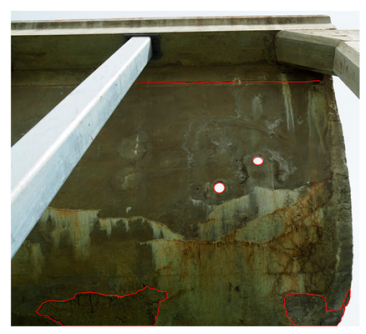

(d)

Figure 12. Flume. $5 \mathrm{~mm}$ point spacing, $C_{\lambda}<0.02$, neighbourhood size $2.5 \mathrm{~cm}$. (a) classical PCA; (b) robust PCA at $\alpha=0.90$; (c) robust PCA at $\alpha=0.75$; (d) is the corresponding digital image.

The results show that $5 \mathrm{~mm}$ scanning resolution allows damage detection. Therefore, for the sake of computational efficiency, the down sampled scanning resolution is preferred to the original, more dense data. Since the size of the smallest damage which can be detected is $1 \mathrm{~cm}$, small damage cannot be detected in PCs with point spacing greater than $5 \mathrm{~mm}$. Comparing damage sizes with neighborhood sizes implies that a $5 \mathrm{~cm}$ neighborhood is not small enough to detect fine details of the 3D structure such as small damage of $1 \mathrm{~cm}$. The reason is that in a large neighborhood each individual 3D point will contribute less to the surface variation estimate. Thus, the spherical neighborhood size should be chosen based on the dimensions of the smallest damage of the structure that must be extracted.

The tests were repeated with robust PCA. Query points removed as outliers are shown in yellow. The data quality from TLS is generally very high, so there are few outliers expected. Moreover, the environment is not dynamic like a construction site, so the percentage of outliers is not expected to be large. Therefore, at first, $\alpha$ is set to 0.75 . The results of the robust PCA tests revealed that $5 \mathrm{~mm}$ resolution, $2.5 \mathrm{~cm}$ neighborhood size and $C_{\lambda}$ threshold of 0.02 provided the most accurate discrimination between damage and non-damage as shown in Figures 9-12. Moreover, it enhances separation of damaged from undamaged conditions compared to the classical tests by discarding some sort of noise and outliers from the data, and thus masking changes caused by data artefacts from changes caused by damage.

The problem with robust PCA is that, in some areas of the structure, such as Figure 11 at $\alpha=0.75$, some damaged points are erroneously flagged as outliers (i.e., detected in yellow). This problem can be dealt with by testing higher percentage of inliers, for example $\alpha=0.80,0.85$, and 0.90 . As shown in Figure 11, at $\alpha=0.90$ the discrimination between damage and outlier classes is improved. 
In Figure 12a, the lines at the connection of the overlapping scans (Figure 13) are classified as non-smooth points (i.e., blue points). Since these erroneous points only appear in the lines at the boundaries of overlapping scans and the other overlapped regions are smooth, this suggests that the problem is not related to registration accuracy, but it is due to the edge effects. The robust PCA can distinguish between data artefacts such as edge effects with damage points and thus removing more of the edge effects as outlier as shown in Figure 12b,c. However, the robust PCA parameters must further be tuned in order to prevent removing of the damaged points erroneously as outliers by applying appropriate percentage of inliers, $\alpha$.
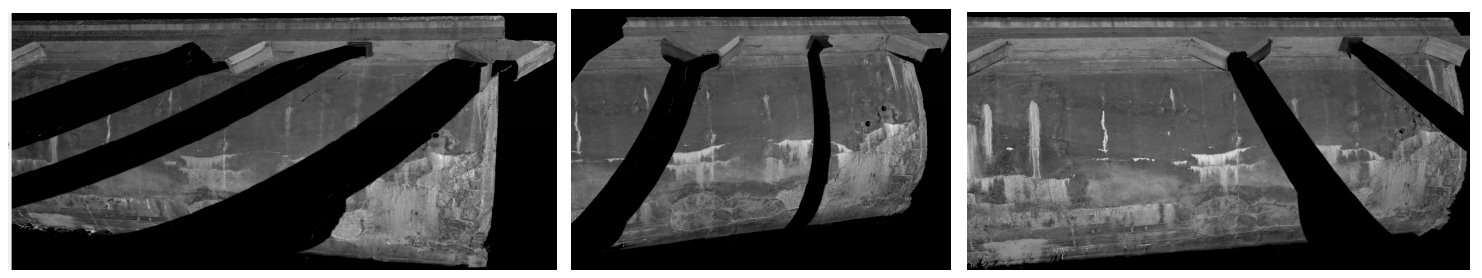

Figure 13. Some of the overlapping scans of the test data.

In the next section, numerical simulations will be applied to investigate the relation between the variables in the experiment based on a systematic and data-independent criterion for shallow damage detection.

\subsection{Systematic Thresholds for Robust PCA Classification}

Different values of robust PCA inlier percentage, $\alpha$, ranging from $65 \%$ to $95 \%$ were tested on different parts of the aqueduct to compute robust $C_{\lambda}$ values at each point. Afterwards, the systematic similarity thresholds derived from Equation (4) were applied to classify the inlier points as damage or non-damage. Figures 14 and 15 show the tests comparing systematic and subjective thresholds while tuning robust PCA inlier percentage for several parts of the aqueduct. Visual inspection of digital imagery of the aqueduct suggests that the classification results defined based on point density the at 0.95 confidence level better identify the damage than the subjective threshold of 0.02 obtained from the initial experiment. 


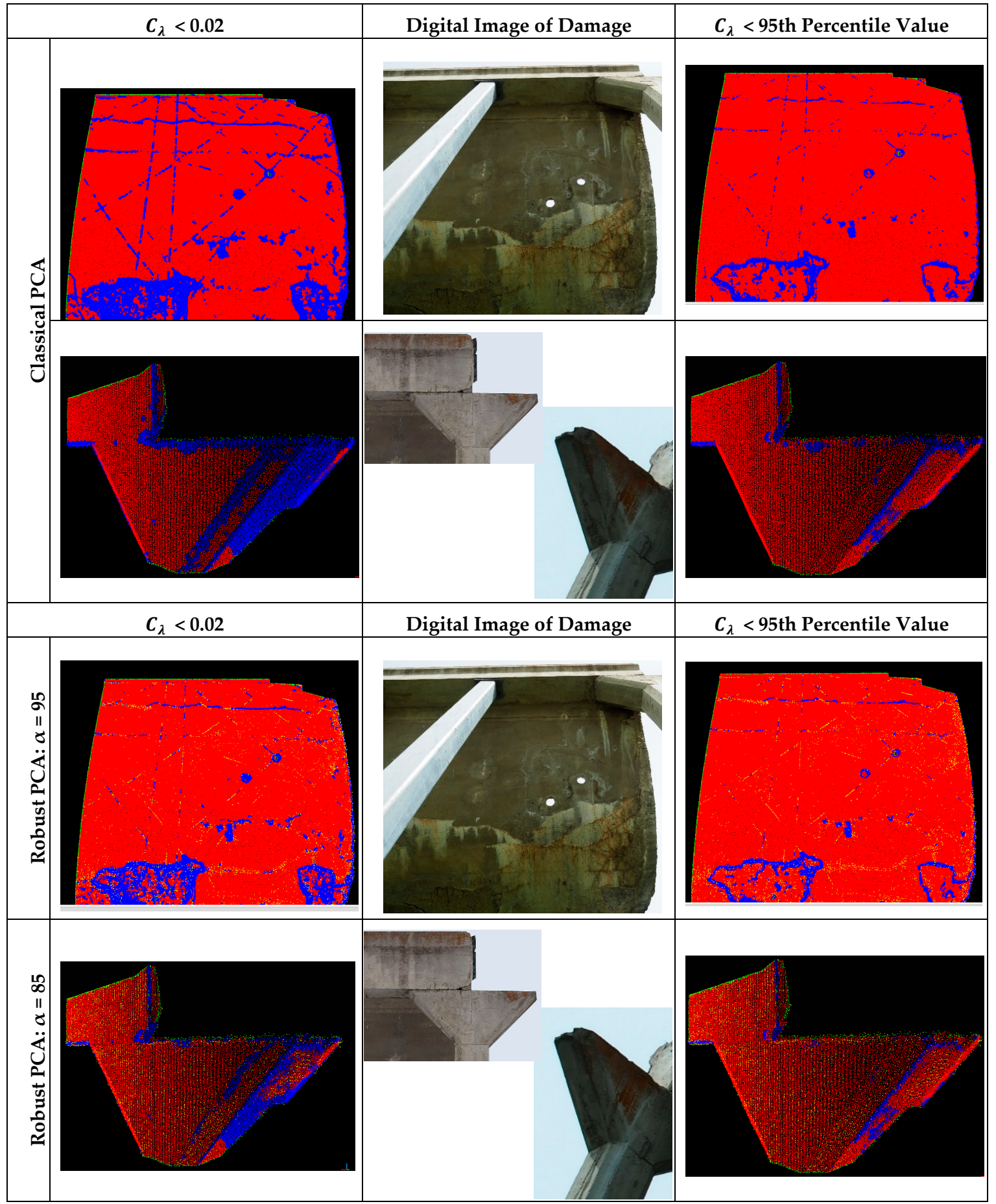

Figure 14. The top of first column and flume. $5 \mathrm{~mm}$ point spacing, neighborhood size $2.5 \mathrm{~cm}$, systematic (right) versus subjective (left) thresholds: classification of damage (blue) and non-damage (red) points after removing outlier points (yellow) at various $\alpha$. 


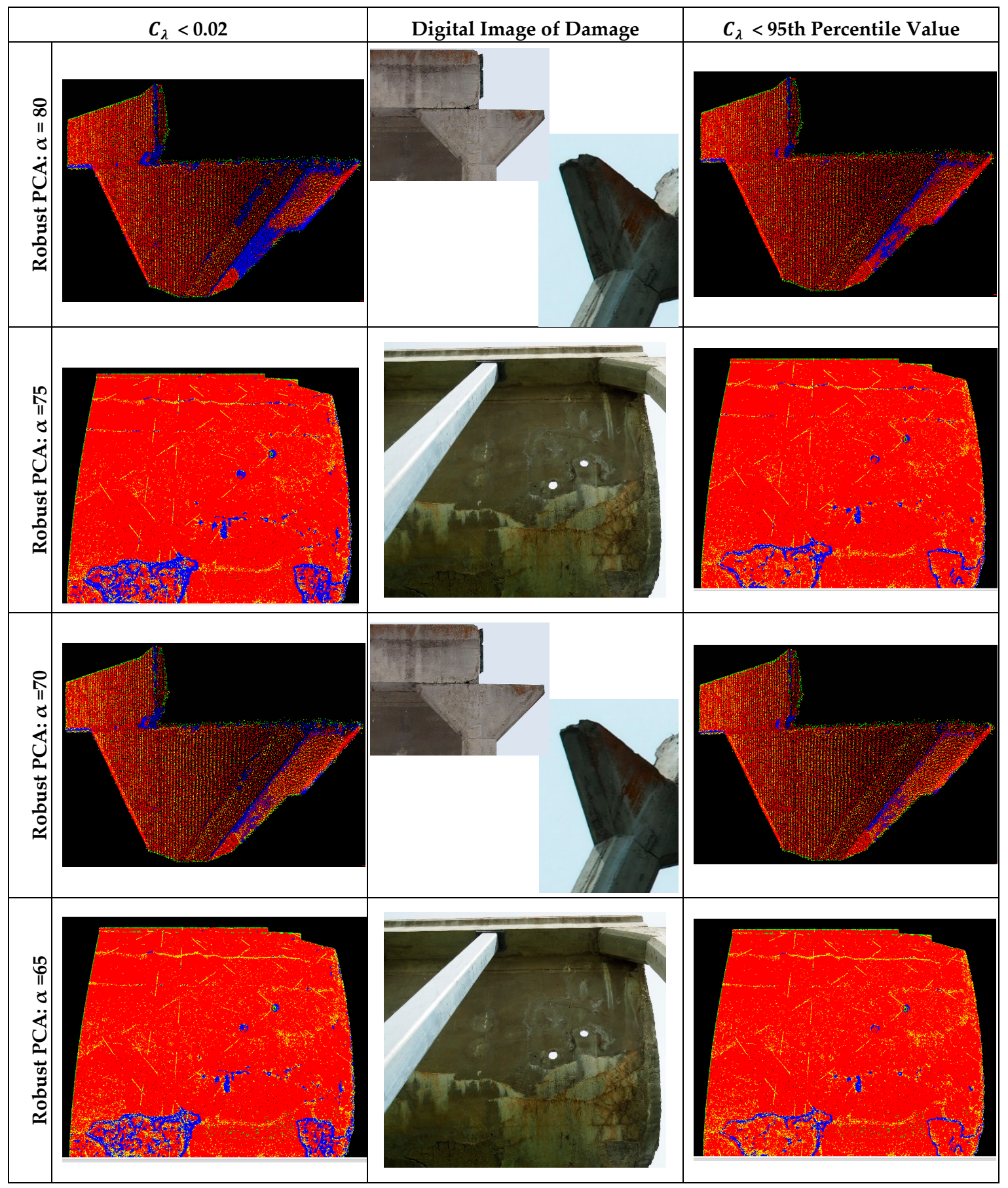

Figure 15. Continuation of Figure 14.

The tests were repeated on a second data set captured of a concrete pedestrian overpass on the University of Calgary campus. The digital imagery in Figures 16 and 17 show the location of the superficial damage. The corresponding classification tests illustrated in Figures 18-20 agree with the aqueduct data set that the simulated thresholds at 0.95 confidence level enhance the damage detection compared to the subjective threshold. 


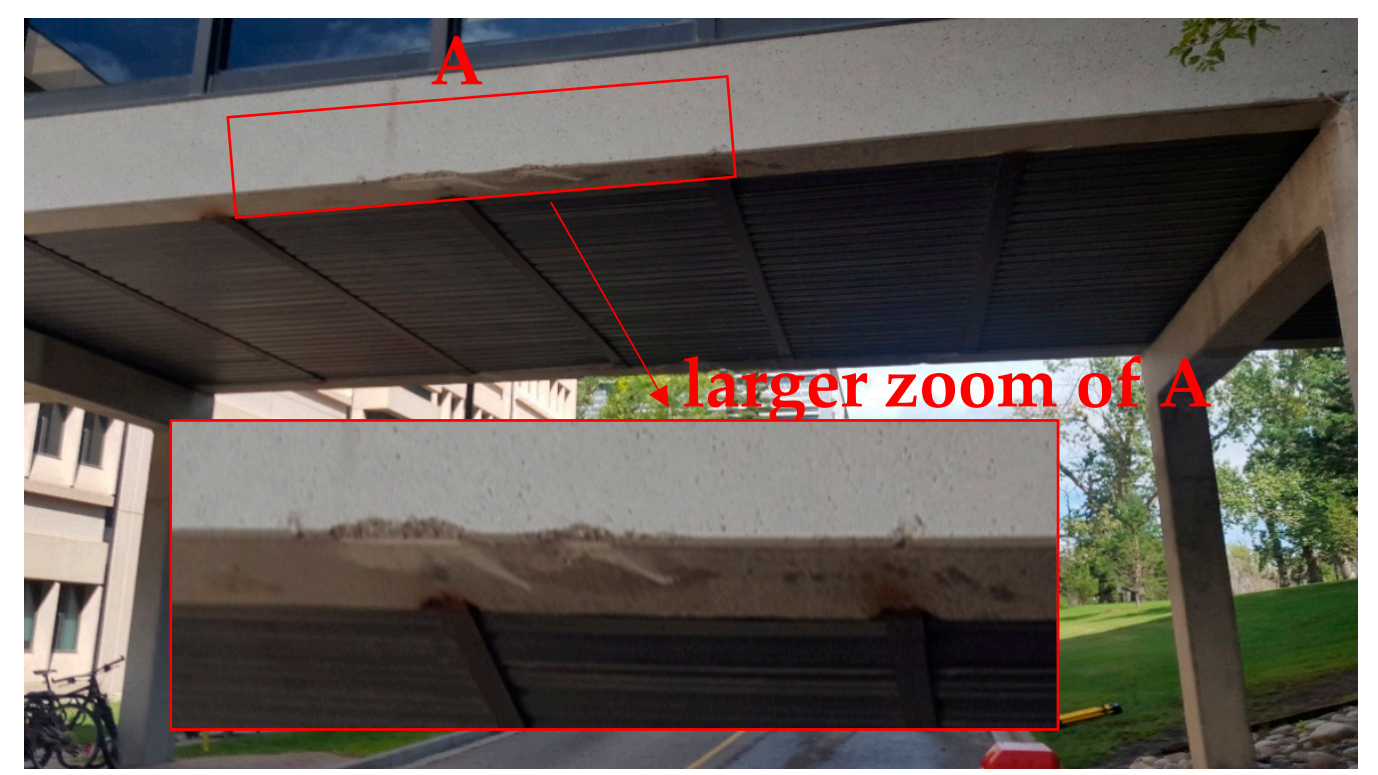

Figure 16. South side of the on-campus pedestrian overpass with visible damage at rectangle area $\mathrm{A}$ and its larger zoom.

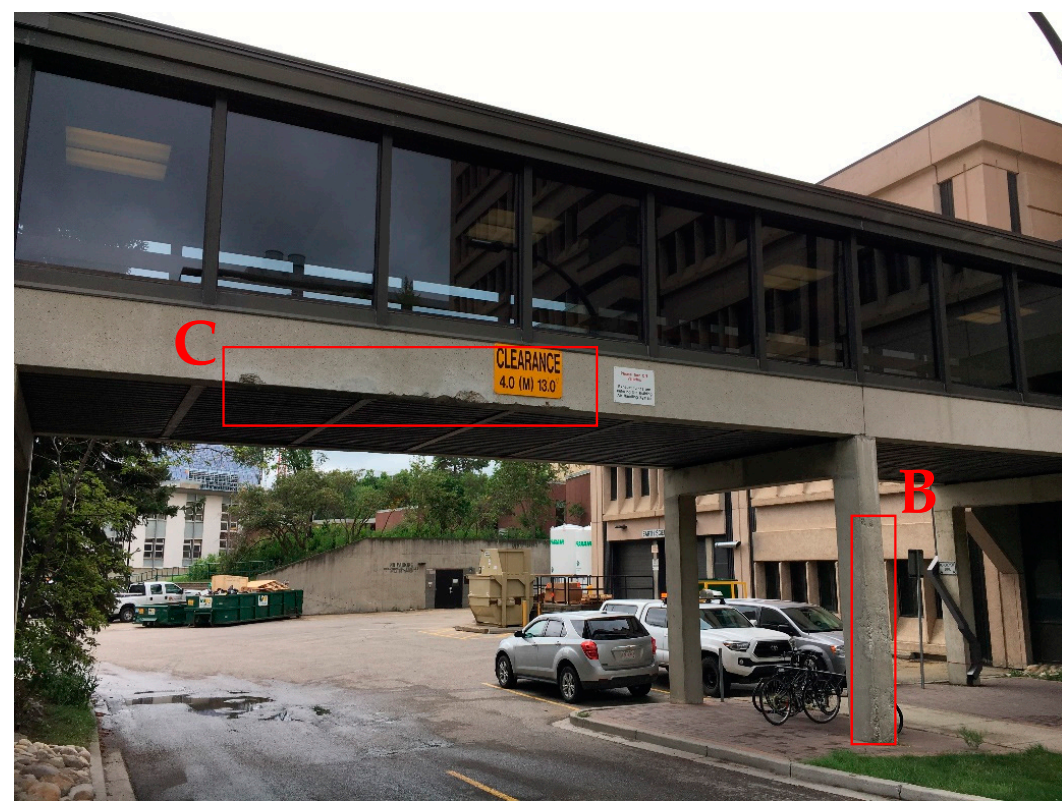

(a)

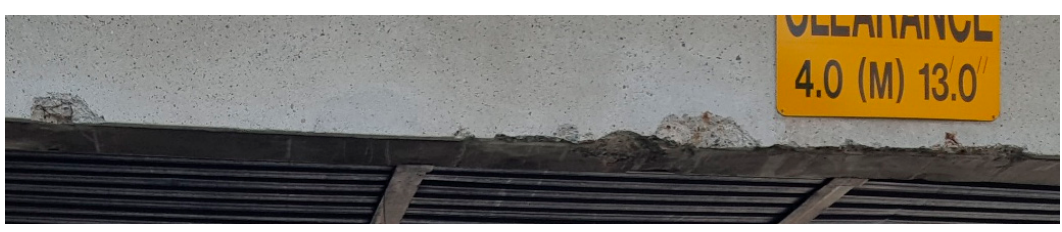

(b)

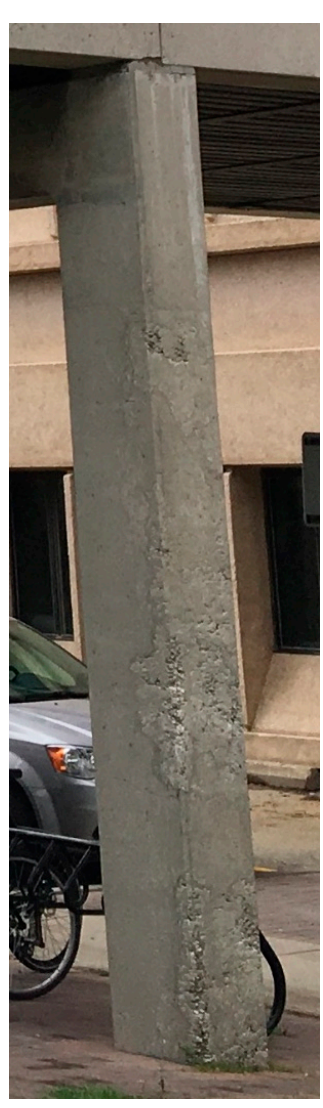

(c)

Figure 17. (a) North side of the on-campus pedestrian overpass with visible damage at rectangle areas $\mathrm{B}$ and $\mathrm{C} ;(\mathbf{b})$ correspond to larger zoom of C in (a); (c) correspond to larger zoom of B in (a). 


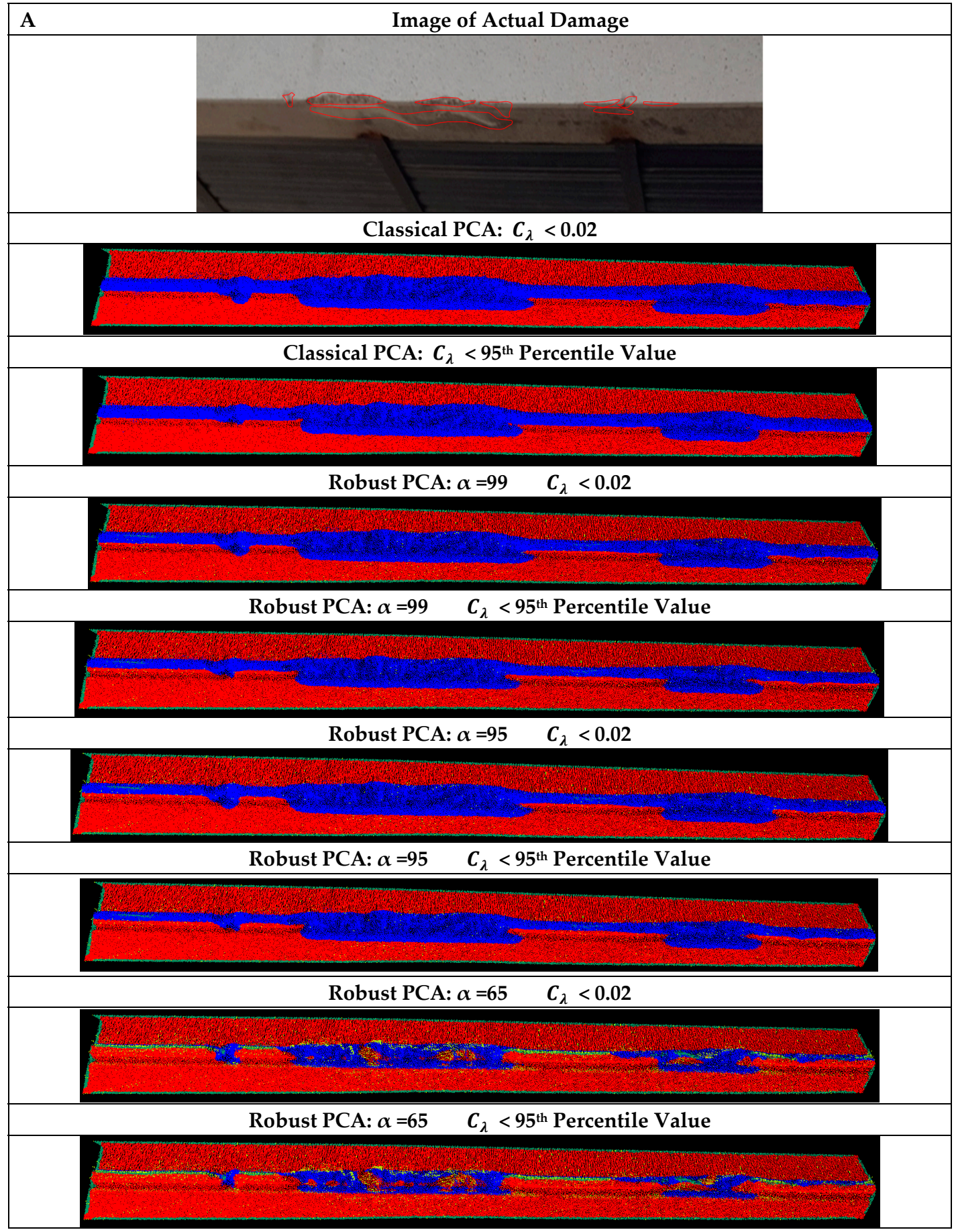

Figure 18. On campus area A. $5 \mathrm{~mm}$ point spacing, neighborhood size $2.5 \mathrm{~cm}$, systematic versus subjective thresholds: classification of damage (blue) and non-damage (red) points after removing outlier points (yellow) at various $\alpha$. 


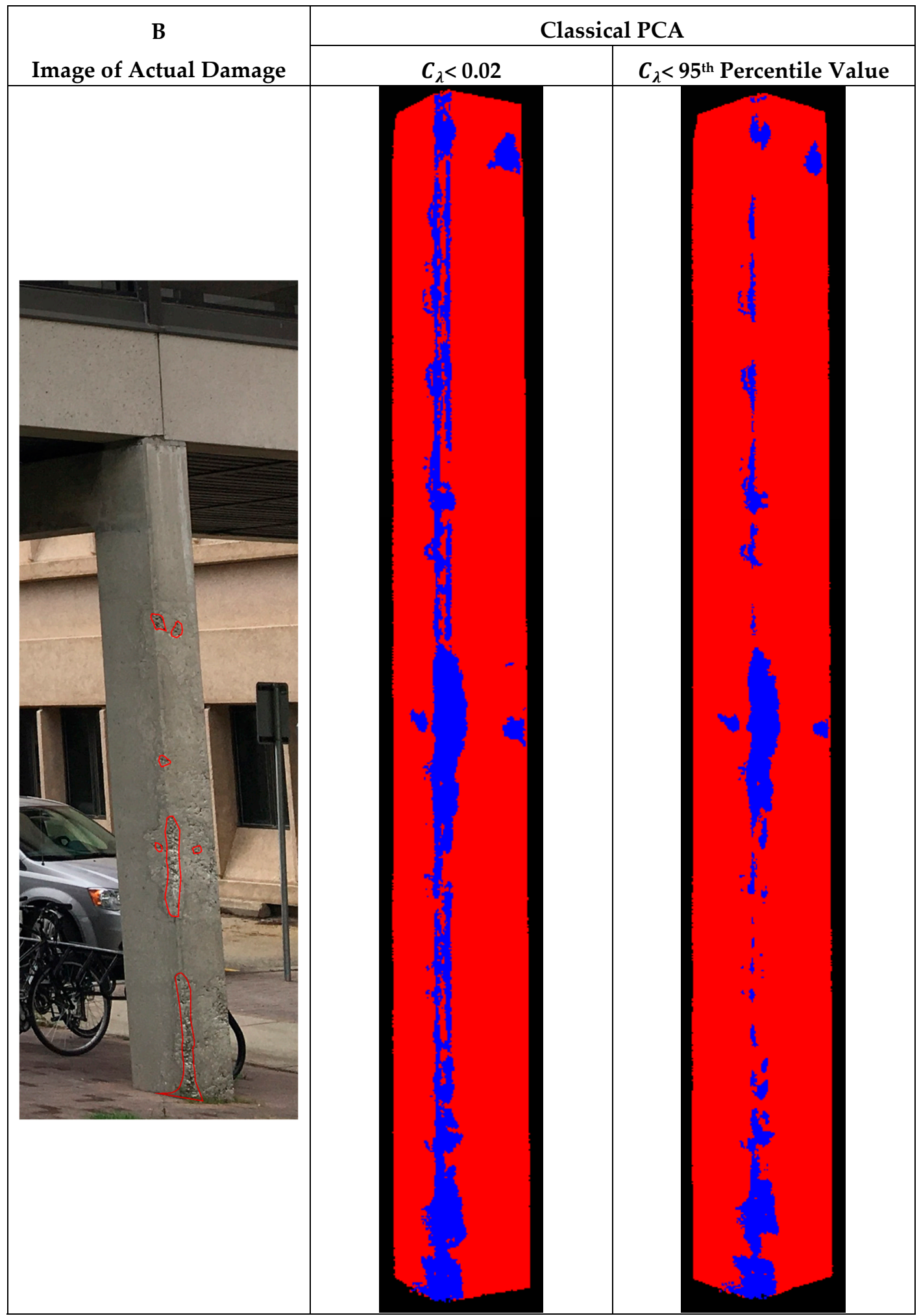

Figure 19. On Campus area B. $5 \mathrm{~mm}$ point spacing, neighborhood size $2.5 \mathrm{~cm}$, systematic versus subjective thresholds: classical PCA classification of damage (blue) and non-damage (red) points. 


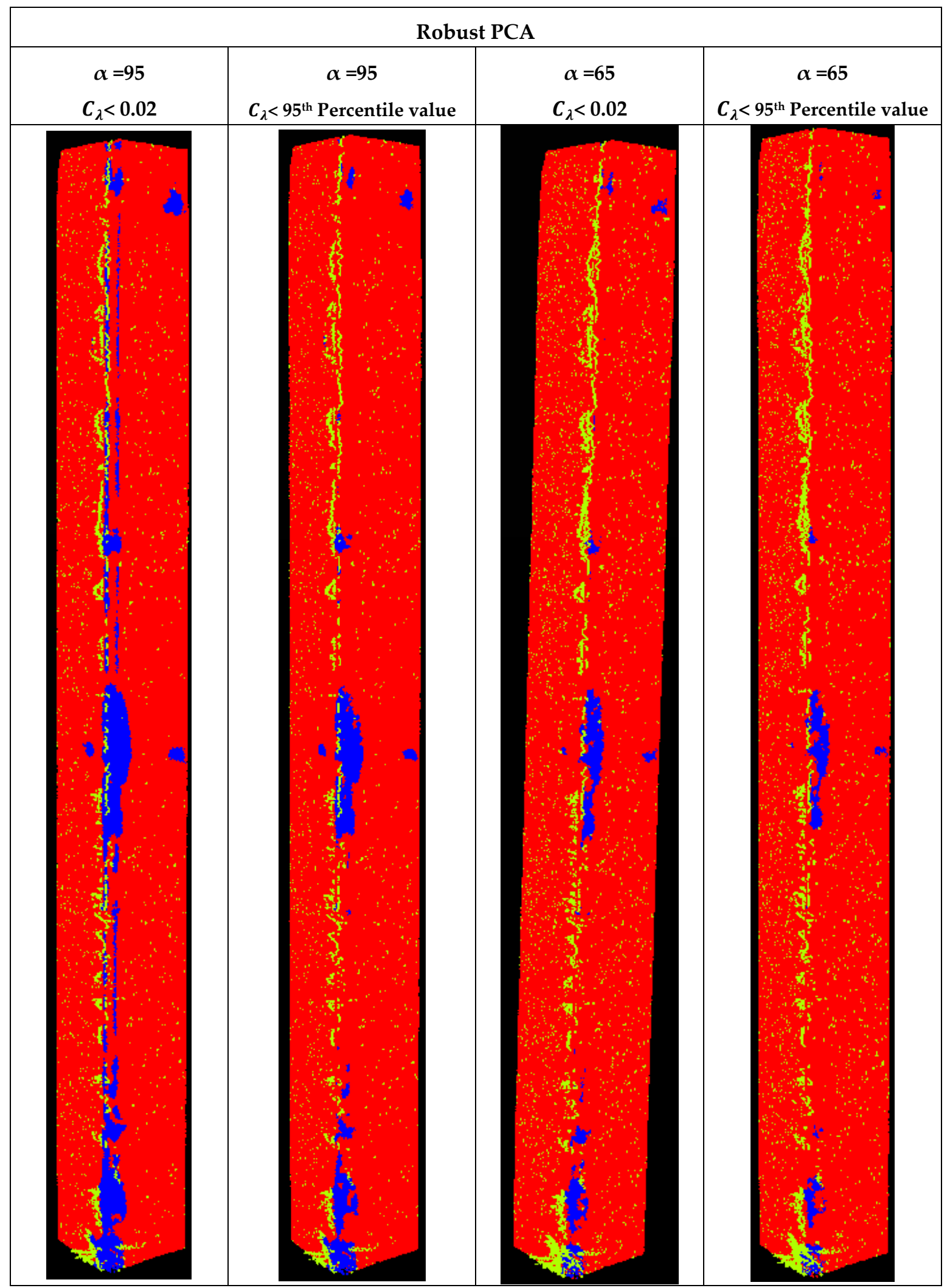

Figure 20. Continuation of Figure 19. Robust PCA classification of damage (blue) and non-damage (red) points after removing outlier points (yellow) at various $\alpha$.

Figure 21, however, shows that neither the systematic nor the subjective thresholds properly detected damage on part $C$ of the second data set. This is evidence that the fixed $2.5 \mathrm{~cm}$ range search 
neighborhood size is not small enough to identify damaged points where the damaged area (e.g., part C) represents surface roughness of less than $1 \mathrm{~cm}$. To detect this damage, smaller neighborhood sizes of $2 \mathrm{~cm}, 1.5 \mathrm{~cm}$, and $1 \mathrm{~cm}$ were tested on part $C$. Moreover, the density higher than $5 \mathrm{~mm}$ point spacing-original (i.e., not-down sampled) $1 \mathrm{~mm}$ scanning resolution-was applied. The reason is that the neighborhood size must be adopted according to the point density by using a larger neighborhood in the areas of lower point density (i.e., higher noise) and a smaller neighborhood in the areas of higher point density (i.e., lower noise).

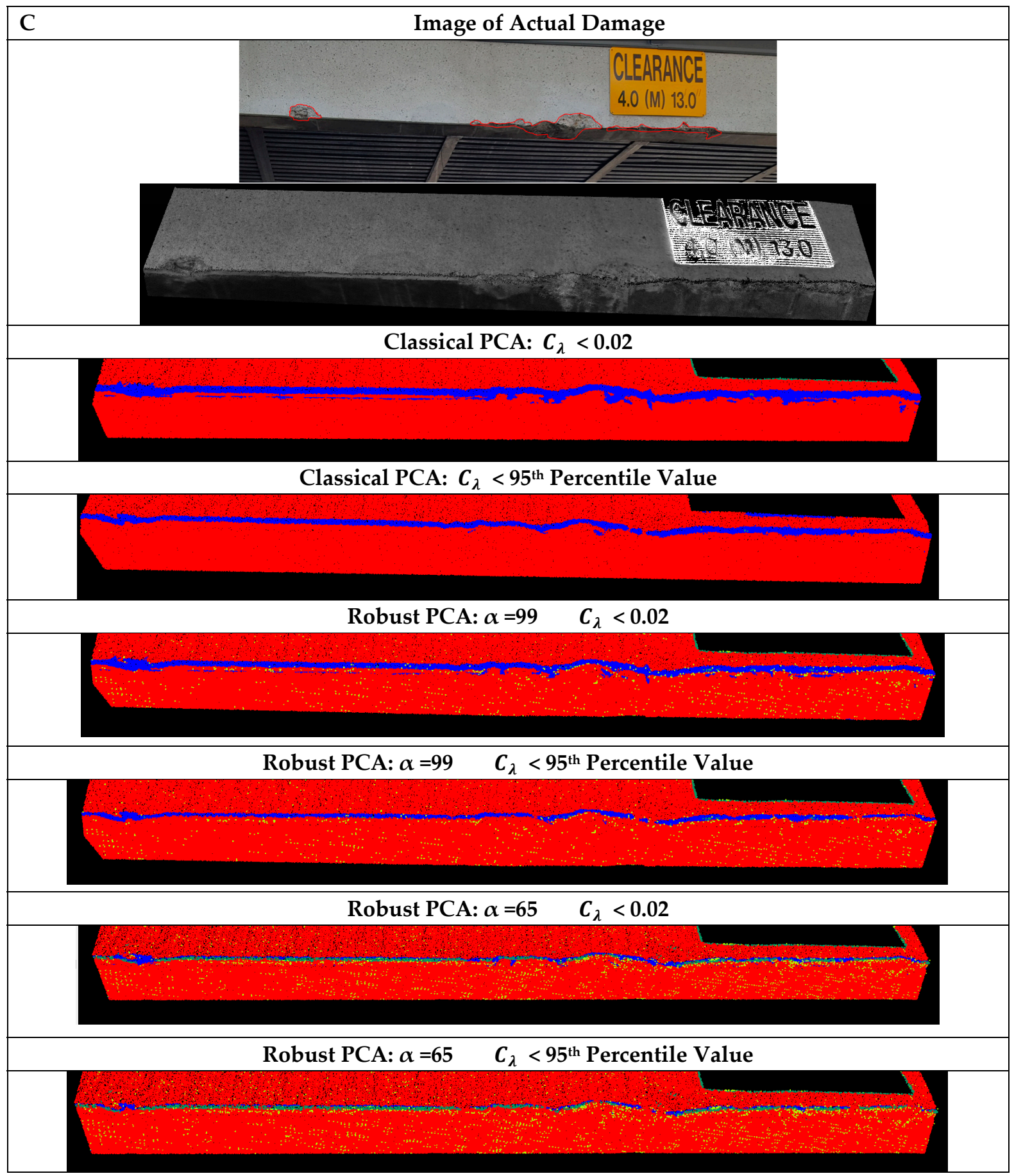

Figure 21. On Campus area C. $5 \mathrm{~mm}$ point spacing, neighborhood size $2.5 \mathrm{~cm}$, systematic versus subjective thresholds: classification of damage (blue) and non-damage (red) points after removing outlier points (yellow) at various $\alpha$. 
As shown in Figure 22, at $1 \mathrm{~mm}$ point spacing and neighborhood size $1 \mathrm{~cm}$, both the systematic threshold and the subjective threshold are able to detect the superficial damaged areas. However, the problem is that some damaged points are erroneously flagged as outliers even at the very large inlier percentage of $\alpha=99$. This can be justified that the very small neighborhood of $1 \mathrm{~cm}$ is not sufficiently large to average out effects of noise and thus robust PCA becomes highly sensitive to noise.

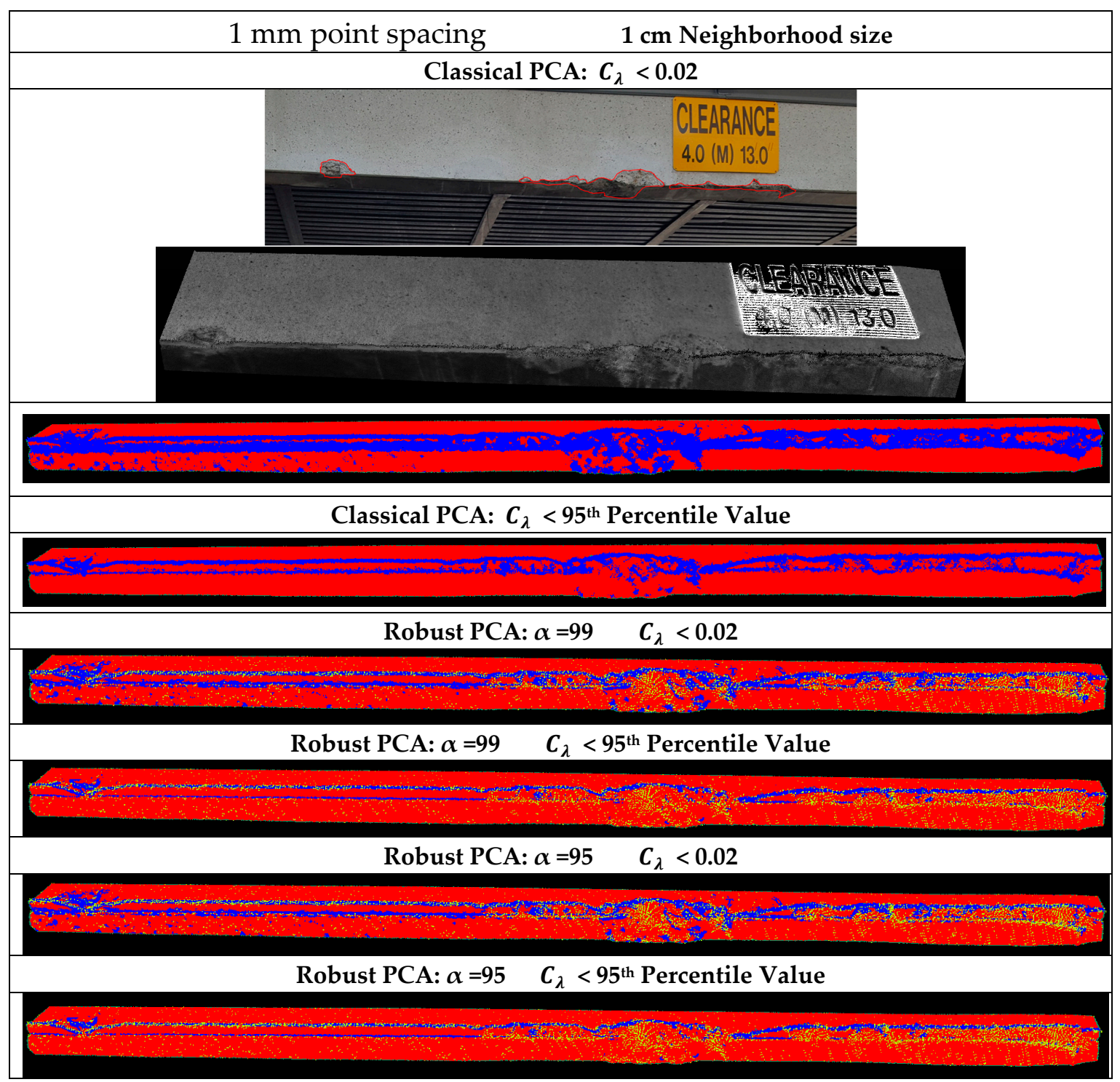

Figure 22. On Campus area C. $1 \mathrm{~mm}$ point spacing (not down sampled), neighborhood size $1 \mathrm{~cm}$, systematic versus subjective thresholds: classification of damage (blue) and non-damage (red) points after removing outlier points (yellow) at various $\alpha$.

\section{Summary, Conclusions and Future Work}

An unsupervised classification algorithm was developed to automatically identify surface damage in TLS point clouds of concrete structures. Based on the experiments, this algorithm requires a minimum density of $5 \mathrm{~mm}$ point spacing and a fixed range search of $2.5 \mathrm{~cm}$ for detecting small damage of $1 \mathrm{~cm}$. When the damaged surface is slightly rough-roughness is smaller than $1 \mathrm{~cm}$-smaller neighborhood size of $1 \mathrm{~cm}$ and density higher than $5 \mathrm{~mm}$ point spacing is required for more accurate detection.

The input to the algorithm is the TLS PC Cartesian coordinates of the surface of the structure. Local surface variation, $C_{\lambda}$, at a fixed range search defined according to the size of smallest damage was selected as a distinguishing and defect-sensitive feature. Robust PCA was used to compute a 
robust estimate of $C_{\lambda}$. The numerical simulations led to the definition of a function to compute a systematic threshold as a function of the number of points encapsulated in the applied fixed range search neighborhood. Accordingly, each 3D point was assigned a semantic label determining whether it is damage or not and visualized in an output scan map. Classification results were validated by comparing manually-delineated damage areas on different perspectives of the available imagery of concrete surface with the relative position of the corresponding defective areas shown on the output map. To validate this method, a synergistic application of different NDT techniques is needed in order to verify the deeper location of damaged parts of the concrete.

The proposed point-wise damage classifier automatically and precisely diagnoses the location of damage of any type, which means roughness as small as $1 \mathrm{~cm}$ or larger occurring on a concrete surface of any geometric shape is captured at any orientation towards the scanner. The algorithm is automated by the systematic threshold definition which quantifies the impact of random errors and density variations within a given fixed range search. This compensates for the optimum neighborhood definition in a more computationally efficient way. The simulated thresholds defined based on point density enhance the damage detection compared to the subjective threshold values, while providing an automatic classification procedure that is not scene dependent. The automated threshold definition based on point density was combined with the robust PCA for outlier detection to achieve more robust geometric features suitable for PC classification of surface damage. However, a consistent and non-subjective value for the most sensitive inlier percentage appropriate for damage detection cannot be suggested for various damage sizes. This is a challenge for robust PCA to suppress noise in TLS PCs while maintaining information about surface flatness.

In the proposed framework, only spatial 3D coordinates, and consequently only geometric features, were required as inputs. Thus, the assumption is that this framework should be general enough to be extendable to other structures which are captured by other types of PCs obtained via dense matching of unmanned aerial vehicle (UAV) imagery, close range imagery or mobile laser scanning, which can provide close range point cloud data with an adequate point density while improving coverage. This assumption will be validated in the future.

Author Contributions: Conceptualization, Zahra Hadavandsiri and Derek D. Lichti; Data curation, Zahra Hadavandsiri, Adam Jahraus and David Jarron; Formal analysis, Zahra Hadavandsiri; Funding acquisition, Derek D. Lichti; Investigation, Zahra Hadavandsiri; Methodology, Zahra Hadavandsiri; Software, Zahra Hadavandsiri; Supervision, Derek D. Lichti; Validation, Zahra Hadavandsiri and Derek D. Lichti; Visualization, Zahra Hadavandsiri; Writing—original draft, Zahra Hadavandsiri; Writing—review \& editing, Zahra Hadavandsiri, Derek D. Lichti, Adam Jahraus and David Jarron.

Funding: This research was funded by the Natural Science and Engineering Research Council of Canada (NSERC) under the grant number of RGPIN-2018-03775.

Acknowledgments: The authors would like to acknowledge the anonymous reviewers for their valuable feedbacks that improve this manuscript.

Conflicts of Interest: The authors declare no conflict of interest.

\section{References}

1. Kumar Mehta, P.; Monteiro, P.J.M. Concrete: Microstructure, Properties, and Materials, 3rd ed.; McGraw-Hill Education Ltd.: New York, NY, USA, 2006.

2. HealCon: Self-Healing Concrete to Create Durable and Sustainable Concrete Structures. EU FP7-NMP, Project ID: 309451, 2013-16. Available online: https://cordis.europa.eu/project/rcn/106380_en.html (accessed on 12 October 2019).

3. Gastineau, A.; Johnson, T.; Schultz, A. Bridge Health Monitoring and Inspections-A Survey of Methods; Transportation Building Report MN/RC; Minnesota Department of Transportation Research Services Section: Saint Paul, MN, USA, 2009; p. 194.

4. Chung, H.W. Assessment and classification of damages in reinforced concrete structures. Concr. Int. 1994, 16, $55-59$. 
5. PCA IS177. Concrete Slab Surface Defects: Causes, Prevention, Repair; Portland Cement Association: Skokie, IL, USA, 2001.

6. Masciotta, M.G.; Roque, J.C.A.; Ramos, L.F.; Lourenço, P.B. A multidisciplinary approach to assess the health state of heritage structures: The case of the Church of Monastery of Jeronimos in Lisbon. Constr. Build. Mater. 2016, 116, 169-187. [CrossRef]

7. Chakraborty, J.; Katunin, A.; Klikowicz, P.; Salamak, M. Early Crack Detection of Reinforced Concrete Structure Using Embedded Sensors. Sensors 2019, 19, 3879. [CrossRef] [PubMed]

8. Kwan, A.K.; Ng, P.L. Building Diagnostic Techniques and Building Diagnosis: The Way Forward. In Engineering Asset Management-Systems, Professional Practices and Certification; Springer: Cham, Germany, 2015; pp. 849-862.

9. Maierhofer, C.; Reinhardt, H.W.; Dobmann, G. (Eds.) Non-Destructive Evaluation of Reinforced Concrete Structures; vol. 1 of Deterioration Processes and Standard Test Methods; Woodhead Publishing: Oxford, UK, 2010.

10. Aktan, A.E.; Farhey, D.N.; Helmicki, A.J.; Brown, D.L.; Hunt, V.J.; Lee, K.L.; Levi, A. Structural Identification for Condition Assessment: Experimental Art. ASCE J. Struct. Eng. 1997, 123, 1674-1684. [CrossRef]

11. Lau, K.-T. Fibre-optic sensors and smart composites for concrete applications. Mag. Concr. Res. 2003, 55, 19-34. [CrossRef]

12. Lu, W.; Teng, J.; Cui, Y. Damage identification for large span structure based on multiscale inputs to artificial neural networks. Sci. World J. 2014, 2014. [CrossRef]

13. Valença, J.; Dias-da-Costa, D.; Gonçalves, L.; Júlio, E.; Araújo, H. Automatic concrete health monitoring: Assessment and monitoring of concrete surfaces. Struct. Infrastruct. Eng. 2014, 10, 1547-1554. [CrossRef]

14. Thomas, J.; Kareem, A.; Bowyer, K. Automated Poststorm Damage Classification of Low-Rise Building Roofing Systems Using High-Resolution Aerial Imagery. IEEE Trans. Geosci. Remote Sens. 2014, 52, 3851-3861. [CrossRef]

15. Jaafar, H.; Meng, X.; Sowter, A. Health monitoring of historic ruins and heritage buildings using terrestrial laser scanning and generalized Procrustes analysis. In Proceedings of the 5th International Conference on Heritage and Sustainable Development, Green Lines Institute, Lisbon, Portugal, 12-15 July 2016.

16. Jenkins, M.D.; Buggy, T.; Morison, G. An Imaging System for Visual Inspection and Structural Condition Monitoring of Railway Tunnels. In Proceedings of the IEEE Workshop on Environmental, Energy, and Structural Monitoring Systems (EESMS), Milan, Italy, 24-25 July 2017.

17. Bayram, B.; Nemli, G.; Özkan, T.; Oflaz, O.E.; Kankotan, B.; Çetin, İ. Comparison of Laser Scanning and Photogrammetry and Their Use for Digital Recording of Cultural Monument Case Study, ISPRS Annals of the Photogrammetry, Remote Sensing and Spatial Information Sciences, Byzantine Land Walls-Istanbul. ISPRS Ann. Photogramm. Remote Sens. Spat. Inf. Sci. 2015, 2, 17. [CrossRef]

18. Ullman, S. The interpretation of structure from motion. Proceedings of the Royal Society of London. Ser. $B$ Biol. Sci. 1979, 203, 405-426.

19. Alfonso-Torreño, A.; Gómez-Gutiérrez, Á.; Schnabel, S.; Contador, J.F.; de Sanjosé Blasco, J.J.; Fernández, M.S. sUAS, SfM-MVS photogrammetry and a topographic algorithm method to quantify the volume of sediments retained in check-dams. Sci. Total Environ. 2019, 678, 369-382. [CrossRef] [PubMed]

20. Giordan, D.; Hayakawa, Y.; Nex, F.; Remondino, F.; Tarolli, P. The use of remotely piloted aircraft systems (RPASs) for natural hazards monitoring and management. Nat. Hazards Earth Syst. Sci. 2018, 18, 1079-1096. [CrossRef]

21. Skarlatos, D.; Kiparissi, S. Comparison of laser scanning, photogrammetry and SFM-MVS pipeline applied in structures and artificial surfaces. ISPRS Ann. Photogramm. Remote Sens. Spat. Inf. Sci. 2012, 3, 299-304. [CrossRef]

22. Walton, G.; Delaloye, D.; Diederichs, M.S. Development of an elliptical fitting algorithm to improve change detection capabilities with applications for deformation monitoring in circular tunnels and shafts. Tunn. Undergr. Space Technol. 2014, 43, 336-349. [CrossRef]

23. Yang, H.; Omidalizarandia, M.; Xu, X.Y.; Neumann, I. Terrestrial laser scanning technology for deformation monitoring and surface modeling of arch structures. Compos. Struct. 2016, 9, 366-369. [CrossRef]

24. Lichti, D.D.; Gordon, S.J.; Stewart, M.P. Ground-based laser scanners: Operations, systems and applications. Geomatica 2002, 56, 21-33. 
25. Valença, J.; Puente, I.; Júlio, E.; González-Jorge, H.; Arias-Sánchez, P. Assessment of cracks on concrete bridges using image processing supported by laser scanning survey. Constr. Build. Mater. 2017, 146, 668-678. [CrossRef]

26. Hancock, C.M.; Roberts, G.W.; Bisby, L.; Cullen, M.; Arbuckle, J. Detecting fire damaged concrete using laser scanning. In Proceedings of the FIG Working Week, Rome, Italy, 6-10 May 2012.

27. Mukupa, W.; Roberts, G.W.; Hancock, C.M.; Al-Manasir, K. A review of the use of terrestrial laser scanning application for change detection and deformation monitoring of structures. Surv. Rev. 2017, 49, 99-116. [CrossRef]

28. Lichti, D.D.; Gordon, S.J.; Tipdecho, T. Error models and propagation in directly georeferenced terrestrial laser scanner networks. J. Surv. Eng. 2005, 131, 135-142. [CrossRef]

29. Vosselman, G.; Maas, H.G. Airborne and Terrestrial Laser Scanning; CRC: Boca Raton, FL, USA, 2010; p. 318.

30. Lindenbergh, R.; Pietrzyk, P. Change detection and deformation analysis using static and mobile laser scanning. Appl. Geomat. 2015, 7, 65-74. [CrossRef]

31. Puente, I.; Lindenbergh, R.; Van Natijne, A.; Esposito, R.; Schipper, R. Monitoring of progressive damage in buildings using laser scan data. Int. Arch. Photogramm. Remote. Sens. Spat. Inf. Sci. ISPRS Arch. 2018, 42, 923-929. [CrossRef]

32. Monserrat, O.; Crosetto, M. Deformation measurement using terrestrial laser scanning data and least squares 3D surface matching. ISPRS J. Photogramm. Remote Sens. 2008, 63, 142-154. [CrossRef]

33. Alba, M.; Fregonese, L.; Prandi, F.; Scaioni, M.; Valgoi, P. Structural monitoring of a large dam by terrestrial laser scanning. Int. Arch. Photogramm. Remote Sens. Spat. Inf. Sci. 2006, 36, 6.

34. Barnhart, T.; Crosby, B. Comparing two methods of surface change detection on an evolving thermokarst using high-temporal-frequency terrestrial laser scanning, Selawik River, Alaska. Remote Sens. 2013, 5 , 2813-2837. [CrossRef]

35. Barsanti, S.G.; Guidi, G.; De Luca, L. Segmentation of 3D Models for Cultural Heritage Structural Analysis-Some Critical Issues. ISPRS Ann. Photogramm. Remote Sens. Spat. Inf. Sci. 2017, 4, 115. [CrossRef]

36. Lague, D.; Brodu, N.; Leroux, J. Accurate 3D comparison of complex topography with terrestrial laser scanner: Application to the Rangitikei canyon (NZ). ISPRS J. Photogramm. Remote Sens. 2013, 82, 10-26. [CrossRef]

37. Kim, M.K.; Sohn, H.; Chang, C.C. Localization and Quantification of Concrete Spalling Defects Using Terrestrial Laser Scanning. J. Comput. Civ. Eng. 2014, 29, 04014086. [CrossRef]

38. Mizoguchi, T.; Koda, Y.; Iwaki, I. Quantitative scaling evaluation of concrete structures based on terrestrial laser scanning. Autom. Constr. 2013, 35, 263-274. [CrossRef]

39. Teza, G.; Galgaro, A.; Moro, F. Contactless recognition of concrete surface damage from laser scanning and curvature computation. NDT E Int. 2009, 42, 240-249. [CrossRef]

40. Chen, S.; Truong-Hong, L.; O'Keeffe, E.; Laefer, D.F.; Mangina, E. Outlier detection of point clouds generating from low cost UAVs for bridge inspection. In Proceedings of the Sixth International Symposium on Life-Cycle Civil Engineering, IALCCE, Ghent, Belgium, 28-31 October 2018.

41. Tang, P.; Huber, D.; Akinci, B. Characterization of laser scanners and algorithms for detecting flatness defects on concrete surfaces. J. Comput. Civ. Eng. 2010, 25, 31-42. [CrossRef]

42. Liu, W.; Chen, S.; Hauser, E. LiDAR-based bridge structure defect detection. Exp. Tech. 2011, 35, $27-34$. [CrossRef]

43. Dittrich, A.; Weinmann, M.; Hinz, S. Analytical and numerical investigations on the accuracy and robustness of geometric features extracted from 3D point cloud data. ISPRS J. Photogramm. Remote Sens. 2017, 126, 195-208. [CrossRef]

44. Santos, A.; Figueiredo, E.; Silva, M.F.; Sales, C.S.; Costa, J.C. Machine learning algorithms for damage detection: Kernel-based approaches. J. Sound Vib. 2016, 363, 584-599. [CrossRef]

45. Schölkopf, B.; Smola, A.; Müller, K.R. Nonlinear component analysis as a kernel eigenvalue problem. Neural Comput. 1998, 10, 1299-1319. [CrossRef]

46. Hoffmann, H. Kernel PCA for novelty detection. Pattern Recognit. 2007, 40, 863-874. [CrossRef]

47. Demantké, J.; Mallet, C.; David, N.; Vallet, B. Dimensionality based scale selection in 3d lidar point clouds. Int. Arch. Photogramm. Remote Sens. Spat. Inf. Sci. 2011, 38, 97-102. [CrossRef] 
48. Niemeyer, J.; Rottensteiner, F.; Soergel, U. Contextual classification of lidar data and building object detection in urban areas. ISPRS J. Photogr. Remote Sens. 2014, 87, 152-165. [CrossRef]

49. Rabbani, T.; Van Den Heuvel, F.A.; Vosselmann, G. Segmentation of point clouds using smoothness constraint. Int. Arch. Photogramm. Remote Sens. Spatial Inf. Sci. 2006, 36, 248-253.

50. Nurunnabi, A.; Belton, D.; West, G. Robust statistical approaches for local planar surface fitting in 3D laser scanning data. ISPRS J. Photogramm. Remote Sens. 2014, 96, 106-122. [CrossRef]

51. Hubert, M.; Rousseeuw, P.J.; Verdonck, T.A. Deterministic algorithm for robust location and scatter. J. Comput. Graph. Stat. 2012, 21, 618-637. [CrossRef]

52. Nurunnabi, A.; West, G.; Belton, D. Robust methods for feature extraction from mobile laser scanning 3D point clouds. Res. Locate 2015, 15, 109-120.

53. Maalek, R.; Lichti, D.D.; Ruwanpura, J.Y. Robust segmentation of planar and linear features of terrestrial laser scanner point clouds acquired from construction sites. Sensors 2018, 18, 819. [CrossRef] [PubMed]

54. Maalek, R.; Lichti, D.D.; Walker, R.; Bhavnani, A.; Ruwanpura, J.Y. Extraction of pipes and flanges from point clouds for automated verification of pre-fabricated modules in oil and gas refinery projects. Autom. Constr. 2019, 103, 150-167. [CrossRef]

55. Mehta, M.L. Random Matrices, 2nd ed.; Academic Press: Boston, MA, USA, 1991.

56. Liu, Y.K. Statistical Behavior of the Eigenvalues of Random Matrices. In Proceedings of the Mathematical Junior Seminar; Princeton University: Princeton, NJ, USA, 2001.

57. Wirtz, T.; Guhr, T. Distribution of the smallest eigenvalue in the correlated Wishart model. Phys. Rev. Lett. 2013, 111, 09410. [CrossRef] [PubMed]

58. Fedoryuk, M.V. Pfaffian Equation. Encyclopedia of Mathematics. Available online: http://www. encyclopediaofmath.org/index.php?title=Pfaffian_equation\&oldid=18964 (accessed on 10 November 2019).

59. Halton, J.H. A combinatorial proof of Cayley's theorem on Pfaffians. J. Comb. Theory 1966, 1, $224-232$. [CrossRef]

60. Edelman, A. Eigenvalues and Condition Numbers of Random Matrices. Ph.D. Thesis, Mathematics Department M.I.T University, Cambridge, MA, USA, 1989.

61. Edelman, A. The distribution and moments of the smallest eigenvalue of a random matrix of Wishart type. Linear Algebra Appl. 1991, 159, 55-80. [CrossRef]

62. Wirtz, T.; Akemann, G.; Guhr, T.; Kieburg, M.; Wegner, R. The smallest eigenvalue distribution in the real Wishart Laguerre ensemble with even topology. J. Phys. A Math. Theor. 2015, 48, 245202. [CrossRef]

63. Mikhail, E.M.; Ackermann, F.E. Observations and Least Squares; Harper and Row: New York, NY, USA, 1976.

64. Manz, D.H.; Robert, E.L. Brooks Aqueduct. Can. J. Civ. Eng. 1989, 16, 684-692. [CrossRef]

65. White, R.T. Brief Historical Review of the Brooks Aqueduct; Eastern Irrigation District Archives and Library: Brooks, Alberta, 1983.

66. Besl, P.J.; Mckay, N.D. A Method for Registration of 3-D Shapes. IEEE Trans. Pattern Anal. Mach. Intell. 1992, 14, 239-256. [CrossRef]

(C) 2019 by the authors. Licensee MDPI, Basel, Switzerland. This article is an open access article distributed under the terms and conditions of the Creative Commons Attribution (CC BY) license (http://creativecommons.org/licenses/by/4.0/). 